\title{
BIFURCATIONS OF PERIODIC SOLUTIONS SATISFYING THE ZERO-HAMILTONIAN CONSTRAINT IN REVERSIBLE DIFFERENTIAL EQUATIONS*
}

\author{
R. E. BEARDMORE ${ }^{\dagger}$, M. A. PELETIER $\ddagger$, C. J. BUDD $§$, AND M. AHMER WADEE
}

\begin{abstract}
This is a study of the existence of bifurcation branches for the problem of finding even, periodic solutions in fourth-order, reversible Hamiltonian systems such that the Hamiltonian evaluates to zero along each solution on the branch. The class considered here is a generalization of both the Swift-Hohenberg and extended Fisher-Kolmogorov equations that have been studied in several recent papers. We obtain the existence of local bifurcations from a trivial solution under mild restrictions on the nonlinearity and obtain existence and disjointness results regarding the global nature of the resulting bifurcating continua for the case where the Hamiltonian has a single-well potential.

The local results rest on two abstract bifurcation theorems which also have applications to sixthorder problems and which show that the curves of zero-Hamiltonian solutions are contained within two-dimensional manifolds of solutions of both negative and positive Hamiltonian.
\end{abstract}

Key words. reversible Hamiltonian systems, Lyapunov-Schmidt reduction

AMS subject classifications. 37G15, 70H33, 70G70, 37C $80,37 \mathrm{C} 30$

DOI. $10.1137 /$ S0036141002418637

1. Introduction. In $[25,7,28,29,36,20,9]$ the authors find periodic solutions of systems of Hamiltonian differential equations with the property of having prescribed zero Hamiltonian. In particular, existence theorems for even periodic orbits satisfying the zero-Hamiltonian constraint in certain fourth-order Hamiltonian systems have been derived by Peletier, Troy, and van den Berg using shooting techniques [35, 36]. While such shooting methods rely heavily on the particular form of the nonlinearities in a given problem and thus suffer from a lack of generality, the techniques do provide a great deal of quantitative information about the solutions. The problem of finding periodic solutions of Hamiltonian systems with prescribed nonzero energy has been studied extensively (see [31, 30] and more recently [4]).

The main contribution of this paper is to view the problem of finding zeroHamiltonian periodic solutions of (1.1) as a one-parameter bifurcation problem from a zero solution, with either period or an external parameter playing the role of bifurcation parameter. To solve this bifurcation problem we formulate two abstract Hopf bifurcation theorems (Theorems 2.2 and 2.4) and deduce the existence of the desired

* Received by the editors November 26, 2002; accepted for publication (in revised form) May 21, 2004; published electronically March 25, 2005. This work was supported by TMR grant ERB 4061 PL 97-0159 on degenerate parabolic partial differential equations and by EPSRC grant GR/L17177 of the Applied Nonlinear Mathematics Initiative.

http://www.siam.org/journals/sima/36-5/41863.html

${ }^{\dagger}$ Department of Mathematics, South Kensington Campus, Imperial College, London SW7 2AZ, United Kingdom (r.beardmore@imperial.ac.uk). The research of this author was supported by Nuffield Foundation Grant NAL/00511/G.

${ }^{\ddagger}$ Centrum voor Wiskunde en Informatica, Kruislaan 413, NL-1098 SJ Amsterdam, The Netherlands (peletier@cwi.nl).

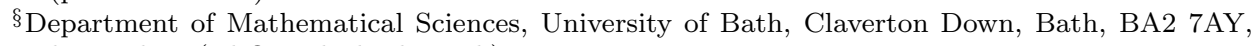
United Kingdom (cjb@maths.bath.ac.uk).

『Department of Civil and Environmental Engineering, South Kensington Campus, Imperial College, London SW7 2AZ, United Kingdom (a.wadee@imperial.ac.uk). 
solutions as a corollary. The abstract results apply to reversible fourth-order Hamiltonian systems at 1:1 and $m: n$ resonances, provided that the Hamiltonian is indefinite about the trivial solution. We call these simple and double bifurcations, respectively, as the theorems lead to either a single continuum or a pair of bifurcating continua of solutions. Furthermore, the proofs of the bifurcation theorems are easily modified to show that the zero-Hamiltonian solutions that we find actually lie within manifolds of solutions of positive and negative Hamiltonian.

The proofs of our abstract results are achieved using a Lyapunov-Schmidt reduction technique, as can be found in many texts [38, 2, 11], and the fact that we essentially have only one bifurcation parameter means that some of the global bifurcation results of [6] are applicable. Using arguments from the configuration-space formulation of fourth-order problems [34, 17, 29], we shall be able to find bifurcation invariants which demonstrate that the bifurcating continua form a countable collection of mutually disjoint sets. Subsequently, we shall be able to show that a simple bifurcation for fourth-order problems results in the existence of an unbounded (in a suitable sense) continuum of solutions, rather like the classical global Hopf bifurcation theorem described in [1]. The global aspect of the paper is peculiar to fourth-order equations and does not immediately apply to more general Hamiltonian systems (like the sixth-order problem [33, equation (2)] for which we also have local results). Consequently, we have what approaches a nonlinear Sturm-Liouville theory (which is well known in the context of elliptic two-point boundary-value problems [5]) for zero-Hamiltonian solutions of (1.1) given below.

A Lyapunov-Schmidt reduction procedure is specifically available for systems that are either reversible or Hamiltonian [37, 22]; however, we do not make use of those results in this paper. The reason for this is that it is not clear that studying the problem in a space of reduced dimension helps to elucidate the role played by the Hamiltonian constraint, and consequently we approach the problem ab initio.

So, consider the class of fourth-order differential equations

$$
u^{\prime \prime \prime \prime}+p u^{\prime \prime}+F_{u}(u)=0,
$$

where primes refer to differentiation with respect to $x, p$ is a real parameter, and the function $F \in C^{\omega}(\mathbb{R})$ satisfies

(F) $F(0)=F_{u}(0)=0$ and $\quad F_{u u}(0)=1$.

Here, $C^{\omega}(\mathbb{R})$ denotes the space of real-analytic functions on $\mathbb{R}$, and a subscript $u$ denotes differentiation with respect to $u$. We shall assume throughout that $F$ satisfies assumption $(\mathbf{F})$ and is therefore positive in some neighborhood of $u=0$. Note that the final condition in $(\mathbf{F})$ is not restrictive as it can always be obtained from a suitable scaling of $u$ and of time (denoted $x$ ), provided that $F_{u u}(0)>0$.

Now (1.1) is reversible (see [8] for a discussion of reversible systems) and Hamiltonian, with Hamiltonian

$$
H \equiv u^{\prime} u^{\prime \prime \prime}-\frac{1}{2} u^{\prime \prime 2}+\frac{1}{2} p u^{\prime 2}+F(u),
$$

and when suitably scaled (see [36]), (1.1) provides the extended Fisher-Kolmogorov and Swift-Hohenberg equations, with $F_{u}(u)= \pm u\left(1-u^{2}\right)$.

Fourth-order equations like (1.1) have a burgeoning literature, as can be seen from the recent studies in $[3,7,24,26,27,28,29,35,36,8,12,19,18]$; see, in particular, the recent monograph [25] and also [36, 20]. In these references it is shown, using a variety of variational, geometric, functional analytic, and elementary techniques, that 
(1.1) may possess periodic, homoclinic, and heteroclinic solutions, with applications ranging from geology to buckling theory; in particular, zero-Hamiltonian periodic solutions play an important role in the study of cellular buckling (see $[12,19,8]$ and the references therein; see also [9]).

We note at this stage that in order for a bifurcation from the trivial solution to occur as $p$ varies, no further restrictions will be required on the nonlinearity $F$ than those already given in assumption $(\mathbf{F})$.

As an application of the results of the first part of the paper, we analyze the behavior of the simple bifurcating branch which connects to $(u, p)=(0,2)$ for the case

$$
F(u ; \epsilon)=\frac{1}{2} u^{2}-\epsilon\left(\frac{1}{4} u^{4}-\frac{1}{6} u^{6}\right),
$$

where $\epsilon$ is a parameter that unfolds the degenerate problem from $\epsilon=0$. In particular, we prove the existence of a fold bifurcation on this bifurcating branch, which was conjectured to exist in [19] and [12]. Finally, the results of some numerical calculations performed in AUTO will be presented, which indicate that similar behavior is observed for the multiple bifurcating branches which connect to the trivial solution at $p>2$. We also compute the symmetry-breaking bifurcations on these branches and illustrate the subsequent connecting branches of solutions.

2. Bifurcation theorems. Let $X, Y$, and $Z$ be Banach spaces, and $B L(X, Y)$ denote the space of continuous (bounded) linear maps from $X$ to $Y$. We write $X^{*}$ for the dual space of continuous linear functionals $B L(X, \mathbb{R})$. If $L \in B L(X, Y)$ and $U \subset X$ is a closed subspace of $X$, then $\left.L\right|_{U} \in B L(U, Y)$ will denote the restriction of $L$ to $U$. We shall use $\|\cdot\|_{X}$ to denote the norm on $X$, and $\operatorname{Iso}(X, Y)$ denotes the set of continuous linear isomorphisms from $X$ to $Y$. Let $C_{2 \pi}^{r}$ be the Banach space of $2 \pi$-periodic $C^{r}$ functions from $[0,2 \pi]$ to $\mathbb{R}^{n}$, endowed with a $C^{r}$ norm.

If $f: X \rightarrow Z$ is a given smooth mapping, then $d f(x)[h]$ will denote the Fréchet derivative of $f$. For higher derivatives, the $k$-form $d^{k} f(x)[h, \ldots, h]$ will also be written as $d^{k} f(x)[h]^{(k)}$ for brevity. Partial derivatives of a function $f \in C^{1}(X \times Y, Z)$ will be written as $d_{x} f(x, y)[h] \in Z$ and $d_{y} f(x, y)[k] \in Z$, where $(h, k) \in X \times Y$, and higher derivatives will be written as in $d_{x y}^{2} f(x, y)[h, k]$. If $X=\mathbb{R}$, we will identify $d_{x} f(x, y)[h]$ with $h d_{x} f(x, y)[1]$, and we shall also write

$$
d^{k} f(x)\left[h_{1}, \ldots, h_{k}\right]=\left(\Pi_{j=1}^{k} h_{j}\right) d^{k} f(x)[1, \ldots, 1],
$$

although we shall often omit the $k$-vector $[1, \ldots, 1]$ in this expression where no confusion results. Given $u, u_{1}, u_{2} \in X$, we will write $\langle u\rangle \equiv \mathbb{R} \cdot u$ and $\left\langle u_{1}, u_{2}\right\rangle=$ $\left\{\alpha_{1} u_{1}+\alpha_{2} u_{2}: \alpha_{1}, \alpha_{2} \in \mathbb{R}\right\}$. For any continuous function $v$, we denote the delta functional by $\delta(v) \equiv v(0)$.

For completeness, let us recall the following. A linear mapping $L \in B L(X, Y)$ is said to be Fredholm if its range $\operatorname{ran}(L)$ is a closed subspace of $Y$ with finite codimension and its null space $\operatorname{ker}(L)$ is a finite-dimensional subspace of $X$. Then

$$
\operatorname{ind}(L)=\operatorname{dim} \operatorname{ker}(L)-\operatorname{codim} \operatorname{ran}(L)
$$

is said to be the Fredholm index of $L$. We recall the following theorem, which gives a useful collections of facts that can be found in [32].

TheOREM 2.1. If $L \in B L(X, Y)$ is Fredholm and $K \in B L(X, Y)$ is a compact linear operator, then $L+K \in B L(X, Y)$ is also Fredholm and $\operatorname{ind}(L+K)=\operatorname{ind}(L)$. 
As a consequence, if $L$ is Fredholm of index zero and is injective, then it is an isomorphism. The set $\mathcal{F}$ of Fredholm operators is open in $B L(X, Y)$, and ind is constant on connected components of $\mathcal{F}$.

2.1. Statement of the abstract problem. Let $\psi \in Z^{*}, M \in C^{\omega}\left(X \times \mathbb{R}^{2}, Y\right)$, and $g \in C^{\omega}\left(X \times \mathbb{R}^{2}, Z\right)$; now consider the bifurcation problem

$$
\mathcal{H}(u, p, \mu) \equiv\left(\begin{array}{c}
M(u, p, \mu) \\
\psi(g(u, p, \mu))
\end{array}\right)=\left(\begin{array}{l}
0 \\
0
\end{array}\right)
$$

where $u \in X$ and $\mu, p \in \mathbb{R}$ are parameters. Throughout, we shall write $\mathcal{H}=M \times \psi(g)$ for brevity. We intend that (2.1) represent an abstract formulation of finding periodic solutions of (1.1) with the property of having zero Hamiltonian; accordingly we shall call the functional $\psi \circ g$ the energy of (2.1). Since the functional $H$ in (1.2) is of quadratic order at the origin, we impose this degree of degeneracy into the operator $g$. Hence we assume that

$$
M(0, p, \mu) \equiv 0
$$

and

$$
g(0, p, \mu) \equiv 0, \quad d_{u} g(0, p, \mu) \equiv 0 .
$$

By the term bifurcation from the trivial solution $u=0$ of $(2.1)$ at $(u, p, \mu)=$ $\left(0, p_{0}, \mu_{0}\right)$ we mean that there is a sequence $\left(u_{n}, p_{n}, \mu_{n}\right) \subset X \backslash\{0\} \times \mathbb{R}^{2}$ which satisfies

$$
\mathcal{H}\left(u_{n}, p_{n}, \mu_{n}\right) \equiv 0, \quad u_{n} \rightarrow 0 \quad \text { and } \quad\left(p_{n}, \mu_{n}\right) \rightarrow\left(p_{0}, \mu_{0}\right) \quad \text { as } n \rightarrow \infty .
$$

2.2. Local bifurcations. Let us now seek conditions under which there is a bifurcation of (2.1) from the trivial solution. The implicit function theorem applied to $(2.1)$ shows that $\left(0, p_{0}, \mu_{0}\right)$ can be a bifurcation point for (2.1) only if

$$
d_{u} M\left(0, p_{0}, \mu_{0}\right) \notin \operatorname{Iso}(X, Y) .
$$

Furthermore, if $M$ is a assumed to be a Fredholm mapping, then a bifurcation can occur only when $d_{u} M\left(0, p_{0}, \mu_{0}\right)$ is not injective. Motivated by this, we shall now consider two such cases:

(i) $\operatorname{dim} \operatorname{ker}\left(d_{u} M\left(0, p_{0}, \mu_{0}\right)\right)=1$,

(ii) $\operatorname{dim} \operatorname{ker}\left(d_{u} M\left(0, p_{0}, \mu_{0}\right)\right)=2$.

Case (i) is reminiscent of the theorem on bifurcation from a simple eigenvalue and will give rise to a unique bifurcating continuum. In case (ii), however, we will be able to locate exactly two distinct bifurcating continua. Let us now proceed with the promised results.

Theorem 2.2 (simple abstract Hopf bifurcation). Suppose that (2.2)-(2.3) hold and that $d_{u} M\left(0, p_{0}, \mu_{0}\right) \in B L(X, Y)$ is Fredholm of index zero, where $\operatorname{ker}\left(d_{u} M\left(0, p_{0}, \mu_{0}\right)\right)$ $=\langle k\rangle$. Suppose also that $X=\langle k\rangle \oplus U, V=\operatorname{ran}\left(d_{u} M\left(0, \mu_{0}, p_{0}\right), Y=\langle K\rangle \oplus V, P\right.$ : $Y \rightarrow V$ is the projection operator along $\langle K\rangle$, and $Q$ is the projection onto $\langle K\rangle$ which is identified with $\mathbb{R}$.

Suppose further that $\psi\left(d_{u u}^{2} g\left(0, p_{0}, \mu_{0}\right)[k, k]\right)=0$ and that the operator $D \in B L(U \times$ $\mathbb{R}^{2}, V \times \mathbb{R}^{2}$ ) given by

$$
D=\left(\begin{array}{ccc}
P d_{u} M\left(0, p_{0}, \mu_{0}\right) & P d_{u p}^{2} M\left(0, p_{0}, \mu_{0}\right)[k, 1] & P d_{u \mu}^{2} M\left(0, p_{0}, \mu_{0}\right)[k, 1] \\
0 & Q d_{u p}^{2} M\left(0, p_{0}, \mu_{0}\right)[k, 1] & Q d_{u \mu}^{2} M\left(0, p_{0}, \mu_{0}\right)[k, 1] \\
\psi\left(d_{u u}^{2} g[k, \cdot]\right) & \psi\left(d_{u u p}^{3} g[k, k, 1]\right) & \psi\left(d_{u u \mu}^{3} g[k, k, 1]\right)
\end{array}\right)
$$


is an isomorphism.

Then, $\left(0, p_{0}, \mu_{0}\right)$ is a bifurcation point for (2.1). Moreover, there is an interval $I$ containing 0 and $a$ unique analytic branch $\mathcal{B}$ of solutions of $(2.1)$ on which $(u, p, \mu)=$ $(u(\beta), p(\beta), \mu(\beta))$ for $\beta \in I$ and which satisfies $u(\beta) \neq 0$ for $\beta \neq 0,(u(0), p(0), \mu(0))=$ $\left(0, p_{0}, \mu_{0}\right)$. Moreover, there results $\|u(\beta)-\beta k\|_{X}=O\left(\beta^{2}\right)$ as $\beta \rightarrow 0$.

Proof. Let us express $u$ in terms of the decomposition of $X$ as $u=\beta k+r=$ $\beta(k+\rho) \in\langle k\rangle \oplus U$; then (2.1) is equivalent to

$$
\begin{aligned}
(P+Q) M(\beta(k+\rho), p, \mu) & =0, \\
\psi(g(\beta(k+\rho), p, \mu)) & =0 .
\end{aligned}
$$

Using analyticity, it follows that there are analytic mappings $\tilde{M}$ and $\tilde{g}$ such that

$$
M(\beta(k+\rho), p, \mu)=\beta d_{u} M(0, p, \mu)[k+\rho]+\beta^{2} \tilde{M}(\beta, \rho, p, \mu)
$$

and

$$
\psi(g(\beta(k+\rho), p, \mu))=\psi\left(\frac{\beta^{2}}{2} d_{u u}^{2} g(0, p, \mu)[k+\rho, k+\rho]+\frac{\beta^{3}}{2} \tilde{g}(\beta, \rho, p, \mu)\right) .
$$

As we are seeking nonzero solutions to (2.1), we can divide by appropriate powers of $\beta$ in (2.5)-(2.6) and solve the equivalent problems

$$
\begin{aligned}
(P+Q) d_{u} M(0, p, \mu)[k+\rho]+\beta \tilde{M}(\beta, \rho, p, \mu) & =0, \\
\psi\left(d_{u u}^{2} g(0, p, \mu)[k+\rho, k+\rho]+\beta \tilde{g}(\beta, \rho, p, \mu)\right) & =0 .
\end{aligned}
$$

In turn, (2.7)-(2.8) is equivalent to

$$
\begin{aligned}
P\left(d_{u} M(0, p, \mu)[k+\rho]+\beta \tilde{M}(\beta, \rho, p, \mu)\right) & =0 \in V, \\
Q\left(d_{u} M(0, p, \mu)[k+\rho]+\beta \tilde{M}(\beta, \rho, p, \mu)\right) & =0 \in \mathbb{R}, \\
\psi\left(d_{u u}^{2} g(0, p, \mu)[k+\rho, k+\rho]+\beta \tilde{g}(\beta, \rho, p, \mu)\right) & =0 \in \mathbb{R},
\end{aligned}
$$

where the one-dimensional space $\langle K\rangle$ is identified with $\mathbb{R}$.

Let us now define $(2.9)-(2.11)$ as $\Phi_{1}(\beta, \rho, p, \mu)=0$, where $\Phi_{1}$ is an analytic mapping of Banach spaces

$$
\Phi_{1}: \mathbb{R} \times U \times \mathbb{R}^{2} \rightarrow V \times \mathbb{R}^{2} .
$$

Under the stated assumptions it is clear that $\Phi_{1}\left(0,0, p_{0}, \mu_{0}\right)=0$, and one can show that $D=d_{\rho, p, \mu} \Phi_{1}\left(0,0, p_{0}, \mu_{0}\right)$, noting $Q\left[d_{u} M\left(0, p_{0}, \mu_{0}\right)\right]=0$ by definition. It now follows by the implicit function theorem that we may locally solve (2.9)-(2.10) for $\rho$, $p$, and $\mu$ as a function of $\beta$. The fact that $\rho(0)=0$ completes the proof.

The following result tells us that Theorem 2.2 is a special case of a more general result which says that the branch $\mathcal{B}$ of zero energy solutions from this theorem is formed from the intersection of a manifold of solutions of $M(u, p, \mu)=0$ with the zero-energy surface $\{(u, p, \mu): \psi(g(u, p, \mu))=0\}$.

TheOREM 2.3. The curve of zero-energy solutions $\mathcal{B}$ from Theorem 2.2 is contained within a (locally) two-dimensional analytic manifold $\mathcal{M}$ of solutions of

$$
M(u, p, \mu)=0
$$

of both positive and negative energy. 
Proof (sketch). Repeat the same argument as in Theorem 2.2 but for the system

$$
\mathcal{H}_{\epsilon}(u, p, \mu) \equiv\left(\begin{array}{c}
M(u, p, \mu) \\
\psi(g(u, p, \mu))-\epsilon
\end{array}\right)=\left(\begin{array}{l}
0 \\
0
\end{array}\right) .
$$

One obtains a mapping $\Phi_{1}(\beta, \rho, p, \mu, \epsilon)$, entirely analogous to (2.12), such that

$$
\Phi_{1}\left(0,0, p_{0}, \mu_{0}, 0\right)=0 \quad \text { and } \quad D=d_{\rho, p, \mu} \Phi_{1}\left(0,0, p_{0}, \mu_{0}, 0\right) .
$$

One can then solve $\rho, p$, and $\mu$ locally as analytic functions of $(\beta, \epsilon)$.

The following technical lemma shows that one can parameterize the bifurcating branch from Theorem 2.2 using one of $p$ or $\mu$ as parameters, and this result will be used at a later stage in the paper.

LEMMA 2.1. If $(u, p, \mu)=(u(\beta), p(\beta), \mu(\beta))$ is an element of the bifurcating branch $\mathcal{B}$ obtained in Theorem 2.2 , then at least one of

$$
d_{u, p}(M \times \psi(g)) \quad \text { or } \quad d_{u, \mu}(M \times \psi(g)) \in B L(X \times \mathbb{R}, Y \times \mathbb{R})
$$

(evaluated at $(u, p, \mu)$ ) is an isomorphism for sufficiently small nonzero $|\beta|$.

Proof. Note from Theorem 2.2 that

$$
\left|Q d_{u p}^{2} M\left(0, p_{0}, \mu_{0}\right)[k, 1]\right|+\left|Q d_{u \mu}^{2} M\left(0, p_{0}, \mu_{0}\right)[k, 1]\right| \neq 0,
$$

and let us therefore assume for definiteness that

$$
Q d_{u p}^{2} M\left(0, p_{0}, \mu_{0}\right)[k, 1] \neq 0 .
$$

Now define the one-parameter family of linear mappings

$$
L(\beta) \equiv\left(\begin{array}{cc}
d_{u} M(u(\beta), p(\beta), \mu(\beta)) & d_{p} M(u(\beta), p(\beta), \mu(\beta)) \\
\psi\left(d_{u} g(u(\beta), p(\beta), \mu(\beta))\right. & \psi\left(d_{p} g(u(\beta), p(\beta), \mu(\beta))\right.
\end{array}\right)
$$

and note that this is an (at most) rank-two perturbation of a Fredholm mapping with index zero. It follows that we need to prove only that $L(\beta)$ is injective for $\beta \neq 0$.

Using analyticity, a straightforward but lengthy calculation shows that we may write $L(\beta)=L_{0}(\beta)+\beta L_{1}(\beta)+\frac{\beta^{2}}{2} L_{2}(\beta)+O\left(\beta^{3}\right)$, where

$$
\begin{gathered}
L_{0}(\beta)=\left(\begin{array}{cc}
d_{u} M & 0 \\
0 & 0
\end{array}\right), \\
L_{1}(\beta)=\left(\begin{array}{cc}
d_{u u}^{2} M[k+O(\beta), \cdot] & d_{u p}^{2} M[k+O(\beta), \cdot] \\
\psi\left(d_{u u}^{2} g[k+O(\beta), \cdot]\right) & 0
\end{array}\right),
\end{gathered}
$$

and

$$
L_{2}(\beta)=\left(\begin{array}{cc}
d_{\text {uuu }}^{3} M[k+O(\beta), k+O(\beta), \cdot] & d_{\text {uup }}^{3} M[k+O(\beta), k+O(\beta), \cdot] \\
\psi\left(d_{\text {uuu }}^{3} g[k+O(\beta), k+O(\beta), \cdot]\right) & \psi\left(d_{\text {uup }}^{3} g[k+O(\beta), k+O(\beta), \cdot]\right)
\end{array}\right),
$$

where each of the given derivatives is evaluated at $(u, p, \mu)=(0, p(\beta), \mu(\beta))$. For $\beta \neq 0$, one can see that $L(\beta)$ is injective if and only if $T(\beta)$ is, where

$$
\begin{aligned}
T(\beta)= & \left(\begin{array}{cc}
d_{u} M & 0 \\
\psi\left(d_{u u}^{2} g[k+O(\beta), \cdot]\right) & 0
\end{array}\right) \\
& +\frac{\beta}{2}\left(\begin{array}{cc}
2 d_{u u}^{2} M[k+O(\beta), \cdot] & 2 d_{u p}^{2} M[k+O(\beta), \cdot] \\
\psi\left(d_{u u u}^{3} g[k+O(\beta), k+O(\beta), \cdot]\right) & \psi\left(d_{u u p}^{3} g[k+O(\beta), k+O(\beta), \cdot]\right)
\end{array}\right) \\
& +O\left(\beta^{2}\right) .
\end{aligned}
$$


Again, each of the derivatives is evaluated at $(u, p, \mu)=(0, p(\beta), \mu(\beta))$.

Clearly $T(0)$ is not injective, but the fact that $d_{u u}^{2} g\left(0, p_{0}, \mu_{0}\right)[k, k] \neq 0$ implies

$$
\operatorname{ker}(T(0))=\langle\kappa\rangle \subset X \times \mathbb{R},
$$

where $\kappa=\left(0_{X}, 1\right) \in X \times \mathbb{R}$. To prove that $T(\beta)$ is injective for small $\beta$ it suffices to prove that

$$
T^{\prime}(0) \kappa \notin \operatorname{ran}(T(0))
$$

However

$$
T^{\prime}(0)=\frac{1}{2}\left(\begin{array}{cc}
2 d_{u u}^{2} M[k, \cdot] & 2 d_{u p}^{2} M[k, \cdot] \\
\psi\left(d_{u u u}^{3} g[k, k, \cdot]\right) & \psi\left(d_{u u p}^{3} g[k, k, \cdot]\right)
\end{array}\right)
$$

evaluating derivatives at $(u, p, \mu)=\left(0, p_{0}, \mu_{0}\right)$, and $\operatorname{ran}(T(0))=\operatorname{ran}\left(d_{u} M\left(0, p_{0}, \mu_{0}\right)\right) \times$ $\operatorname{ran}\left(\psi\left(d_{u u}^{2} g\left(0, p_{0}, \mu_{0}\right)\right)\right)$. It follows that $T^{\prime}(0) \kappa \in \operatorname{ran}(T(0))$ can be satisfied only if

$$
d_{u p}^{2} M\left(0, p_{0}, \mu_{0}\right)[k, 1] \in \operatorname{ran}\left(d_{u} M\left(0, p_{0}, \mu_{0}\right)\right),
$$

but this contradicts (2.15). Finally, one can use an analogous argument to cover the case whereby $Q d_{u \mu}^{2} M\left(0, p_{0}, \mu_{0}\right)[k, 1] \neq 0$.

Next we consider case (ii), where $d_{u} M\left(0, p_{0}, \mu_{0}\right)$ has a two-dimensional null-space.

TheOREM 2.4 (double abstract Hopf bifurcation). Suppose that (2.2)-(2.3) hold and $\operatorname{ker}\left(d_{u} M\left(0, p_{0}, \mu_{0}\right)\right)=W$, where $\operatorname{dim}(W)=2$ with $W=\left\langle k_{1}, k_{2}\right\rangle$. Suppose further that $X=W \oplus U$ and

$$
Y=Z \oplus V, \quad V=\operatorname{ran}\left(d_{u} H\left(0, p_{0}, \mu_{0}\right)\right)
$$

where $V$ is closed, $\operatorname{dim}(Z)=2$, and $Z=\left\langle u_{1}, u_{2}\right\rangle$.

Now, let $P: Y \rightarrow V$ be the projection along $Z$ and $Q=I-P$. For $i=1,2$, let $Q_{i}$ be the projection of $Y$ onto $\left\langle u_{i}\right\rangle$ (which we identify with $\mathbb{R}$ ) such that $Q[y]=$ $Q_{1}[y] u_{1}+Q_{2}[y] u_{2}$. Set

$$
A=\psi\left(d_{u u}^{2} g\left(0, p_{0}, \mu_{0}\right)\left[k_{2}, k_{2}\right]\right), \quad B=\psi\left(d_{u u}^{2} g\left(0, p_{0}, \mu_{0}\right)\left[k_{1}, k_{2}\right]\right)
$$

and

$$
C=\psi\left(d_{u u}^{2} g\left(0, p_{0}, \mu_{0}\right)\left[k_{1}, k_{1}\right]\right) .
$$

Suppose that $C \neq 0, B^{2}>A C$, and let $\alpha_{ \pm}$be the two (real nonzero distinct) roots of the quadratic equation $A \alpha^{2}+2 B \alpha+C=0$. Suppose also that

$$
\operatorname{det}\left(\begin{array}{cc}
Q_{1} d_{u p}^{2} M\left[k_{1}+\alpha_{ \pm} k_{2}, 1\right] & Q_{1} d_{u \mu}^{2} M\left[k_{1}+\alpha_{ \pm} k_{2}, 1\right] \\
Q_{2} d_{u p}^{2} M\left[k_{1}+\alpha_{ \pm} k_{2}, 1\right] & Q_{2} d_{u \mu}^{2} M\left[k_{1}+\alpha_{ \pm} k_{2}, 1\right]
\end{array}\right) \neq 0
$$

when $(u, p, \mu)=\left(0, p_{0}, \mu_{0}\right)$.

Then $\left(0, p_{0}, \mu_{0}\right)$ is a bifurcation point for (2.1). Moreover, there is an interval $I$ containing 0 and exactly two analytic branches $\mathcal{B}_{ \pm}$of solutions of (2.1) on which $(u, p, \mu)=\left(u_{ \pm}(\beta), p_{ \pm}(\beta), \mu_{ \pm}(\beta)\right)$ for $\beta \in I$, with $u_{ \pm}(0) \neq 0$ for $\beta \neq 0$ and $\left(u_{ \pm}(0), p_{ \pm}(0), \mu_{ \pm}(0)\right)=\left(0, p_{0}, \mu_{0}\right)$. Moreover, there are analytic functions $\alpha_{ \pm}: I \rightarrow \mathbb{R}$ and $\rho: I \rightarrow V$ such that $\alpha_{ \pm}(0)=\alpha_{ \pm},\|\rho(\beta)\|_{Y}=O(\beta)$ as $\beta \rightarrow 0$, and $u_{ \pm}(\beta)=$ $\beta k_{1}+\beta \alpha_{ \pm}(\beta) k_{2}+\beta \rho(\beta)$. 
Proof. Using the analyticity of $M$, let us write

$$
M(u, p, \mu)=d_{u} M(0, p, \mu)[u]+\mathcal{O}(2)
$$

and

$$
g(u, p, \mu)=g(0, p, \mu)+d_{u} g(0, p, \mu) u+\frac{1}{2} d_{u u}^{2} g(0, p, \mu)[u, u]+\mathcal{O}(3),
$$

where $\mathcal{O}(n)$ represents any function, $\Theta(u, p, \mu)$ say, where there is a $\gamma>0$ such that $\|\Theta(u, p, \mu)\| \leq \gamma\|u\|^{n}$ for all $(u, p, \mu)$ in a neighborhood of $\left(0, p_{0}, \mu_{0}\right)$.

Now let $u=\beta\left(k_{1}+\alpha k_{2}+\alpha \rho\right) \in W \oplus U$, and note that there is an analytic function $\phi_{1}$ such that the equation $M(u, p, \mu)=0$ is locally equivalent to

$$
\beta d_{u} M(0, p, \mu)\left[k_{1}+\alpha k_{2}+\alpha \rho\right]+\beta^{2} \phi_{1}(\alpha, \beta, \rho, p, \mu)=0 .
$$

We may also use the analyticity of $g$ to write

$$
g\left(\beta\left(k_{1}+\alpha k_{2}+\alpha \rho\right), p, \mu\right)=\frac{\beta^{2}}{2} d_{u u}^{2} g(0, p, \mu)\left[k_{1}+\alpha k_{2}+\alpha \rho\right]^{(2)}+\frac{\beta^{3}}{2} \phi_{2}(\alpha, \beta, \rho, p, \mu),
$$

where $\phi_{2}$ is another suitably defined analytic function. Now the equation $M(u, p, \mu)=$ 0 is equivalent to

$$
\left(P+u_{1} Q_{1}+u_{2} Q_{2}\right) M(u, p, \mu)=0,
$$

and, after dividing (2.17) and (2.18) by $\beta$ and $\beta^{2}$, respectively, we obtain the locally equivalent problem

$$
\begin{aligned}
P\left[d_{u} M(0, p, \mu)\left[k_{1}+\alpha k_{2}+\alpha \rho\right]+\beta \phi_{1}(\alpha, \beta, \rho, p, \mu)\right] & =0 \in V, \\
Q_{1}\left[d_{u} M(0, p, \mu)\left[k_{1}+\alpha k_{2}+\alpha \rho\right]+\beta \phi_{1}(\alpha, \beta, \rho, p, \mu)\right] & =0 \in \mathbb{R}, \\
Q_{2}\left[d_{u} M(0, p, \mu)\left[k_{1}+\alpha k_{2}+\alpha \rho\right]+\beta \phi_{1}(\alpha, \beta, \rho, p, \mu)\right] & =0 \in \mathbb{R}, \\
\psi\left(d_{u u}^{2} g(0, p, \mu)\left[k_{1}+\alpha k_{2}+\alpha \rho\right]\right. & \left.(2)+\beta \phi_{2}(\alpha, \beta, \rho, p, \mu)\right)=0 \in \mathbb{R} .
\end{aligned}
$$

Setting $\beta=0, \rho=0, p=p_{0}$, and $\mu=\mu_{0}$ in (2.19)-(2.22), we find an equation for $\alpha$ :

$$
\psi\left(d_{u u}^{2} g(0, p, \mu)\left[k_{1}+\alpha k_{2}, k_{1}+\alpha k_{2}\right]\right)=0 .
$$

From the definitions of $A, B$, and $C$ given in the statement of the theorem, (2.23) is simply the equation $C+2 B \alpha+A \alpha^{2}=0$ with solutions $\alpha=\alpha_{ \pm}$.

We now write $(2.19)-(2.22)$ as $\Phi_{2}(\rho, p, \mu, \alpha, \beta)=0$, say, where $\Phi_{2}$ is an analytic mapping $\Phi_{2}: U \times \mathbb{R}^{4} \rightarrow V \times \mathbb{R}^{3}$. The derivative $d_{\rho, p, \mu, \alpha} \Phi_{2}\left(0, p_{0}, \mu_{0}, \alpha_{ \pm}, 0\right)$ is given by the operator matrix

$$
L \equiv\left(\begin{array}{ccc}
A_{0} & B_{0} & 0 \\
0 & D_{0} & 0 \\
E_{0} & F_{0} & G_{0}
\end{array}\right) \in B L\left(U \times \mathbb{R}^{3}, V \times \mathbb{R}^{3}\right),
$$

where

$$
A_{0}=\alpha_{ \pm} P d_{u} M^{0}, \quad B_{0}=\left[P d_{u p}^{2} M^{0}\left[k_{1}+\alpha_{ \pm} k_{2}, 1\right] \mid P d_{u \mu}^{2} M^{0}\left[k_{1}+\alpha_{ \pm} k_{2}, 1\right]\right]
$$




$$
\begin{gathered}
E_{0}=2 \alpha_{ \pm} \psi\left(d_{u u}^{2} g^{0}\left[\cdot, k_{1}+\alpha_{ \pm} k_{2}\right]\right), \quad G_{0}=2 \psi\left(d_{u u}^{2} g^{0}\left[k_{2}, k_{1}+\alpha_{ \pm} k_{2}\right]\right) \\
F_{0}=\left[\psi\left(d_{u u p}^{3} g^{0}\left[1, k_{1}+\alpha_{ \pm} k_{2}, k_{1}+\alpha_{ \pm} k_{2}\right]\right) \mid \psi\left(d_{u u \mu}^{3} g^{0}\left[1, k_{1}+\alpha_{ \pm} k_{2}, k_{1}+\alpha_{ \pm} k_{2}\right]\right)\right]
\end{gathered}
$$

and

$$
D_{0}=\left(\begin{array}{cc}
Q_{1} d_{u p}^{2} M^{0}\left[k_{1}+\alpha_{ \pm} k_{2}, 1\right] & Q_{1} d_{u \mu}^{2} M^{0}\left[k_{1}+\alpha_{ \pm} k_{2}, 1\right] \\
Q_{2} d_{u p}^{2} M^{0}\left[k_{1}+\alpha_{ \pm} k_{2}, 1\right] & Q_{2} d_{u \mu}^{2} M^{0}\left[k_{1}+\alpha_{ \pm} k_{2}, 1\right]
\end{array}\right)
$$

and a superscript zero $\left({ }^{0}\right)$ denotes evaluation of a function at $(u, p, \mu)=\left(0, p_{0}, \mu_{0}\right)$.

Clearly, for $L$ to be an isomorphism we require $G_{0} \neq 0$, that is, $\psi\left(d_{u u}^{2} g^{0}\left[k_{2}, k_{1}+\right.\right.$ $\left.\left.\alpha_{ \pm} k_{2}\right]\right) \neq 0$, but this is just $B+\alpha_{ \pm} A \neq 0$, which is true by assumption. Since $A_{0} \in \operatorname{Iso}(U, V), L$ is an isomorphism if $\operatorname{det}\left(D_{0}\right) \neq 0$, and this is also an assumption. Using the implicit function theorem, we can now determine all of the variables as analytic functions of $\beta$ locally to the two points $(\rho, p, \mu, \alpha, \beta)=$ $\left(0, p_{0}, \mu_{0}, \alpha_{ \pm}, 0\right)$.

As was the case for Theorems 2.2 and 2.3, we can prove that the branches of zeroenergy solutions $\mathcal{B}_{ \pm}$from Theorem 2.4 are obtained from the intersection of solutions of $M(u, p, \mu)=0$ with the zero-energy surface.

THEOREM 2.5. The two curves of zero-energy solutions $\mathcal{B}_{ \pm}$from Theorem 2.4 are each contained within (locally) two-dimensional analytic manifolds $\mathcal{M}_{ \pm}$of solutions of $M(u, p, \mu)=0$ of both positive and negative energy. Moreover, $\mathcal{M}_{+} \cap \mathcal{M}_{-}=$ $\left\{\left(0, p_{0}, \mu_{0}\right)\right\}$.

Proof. This is almost a verbatim repetition of the proof of Theorem 2.4, but modified to deal with an energy constraint of the form $\psi(g(u, p, \mu))=\epsilon$.

\section{The existence of bifurcations for fourth- and sixth-order systems.}

3.1. Preliminaries. In this section we shall apply Theorems 2.1 and 2.2 to find bifurcating branches of periodic solutions of (1.1) which have the zero-Hamiltonian property. To do so, we shall presume that a periodic solution of (1.1) has period $T$, where

$$
T=\frac{2 \pi}{\mu}
$$

and $\mu$ is a priori unknown. Upon setting

$$
t=\mu x
$$

a simple rescaling of (1.1) and (1.2) leads us to consider the two-parameter problem

$$
\begin{array}{r}
M(u, p, \mu) \equiv \mu^{4} u^{\prime \prime \prime \prime}+p \mu^{2} u^{\prime \prime}+F_{u}(u)=0, \\
\psi(g(u, p, \mu)) \equiv \delta\left(\mu^{4} u^{\prime} u^{\prime \prime \prime}-\frac{1}{2} \mu^{4} u^{\prime \prime 2}+\frac{1}{2} p \mu^{2} u^{\prime 2}+F(u)\right)=0,
\end{array}
$$

where primes now denote differentiation with respect to $t$. With regard to $(2.1), \psi$ corresponds to $\delta$, and $g$ is the Hamiltonian which appears in (3.2); again we shall write $\mathcal{H}=M \times \psi(g)$ so that (3.1)-(3.2) corresponds to the equation $\mathcal{H}=0$.

A natural setting for the application of these Theorems 2.1 and 2.2 is in the space of even functions of period $2 \pi$. Accordingly, let $X_{e}=\left\{u \in C_{2 \pi}^{4}: u(t)=u(-t)\right\}$ and 
$Y_{e}=\left\{u \in C_{2 \pi}^{0}: u(t)=u(-t)\right\}$, both endowed with their usual norms. We also define the even-odd subspaces

$$
X_{e o}=\left\{u \in X_{e}: u\left(\frac{\pi}{2}-t\right)=-u\left(\frac{\pi}{2}+t\right)\right\}
$$

and

$$
Y_{e o}=\left\{u \in Y_{e}: u\left(\frac{\pi}{2}-t\right)=-u\left(\frac{\pi}{2}+t\right)\right\}
$$

For a given subspace $S \subset Z \subset L^{2}(0, \pi)$ we define its orthogonal complement by $S^{\perp}=\left\{u \in Z: \int_{0}^{2 \pi} u(t) s(t) d t=0 \forall s \in S\right\}$. In this way we obtain a map $\mathcal{H} \in C^{\omega}\left(X_{e} \times\right.$ $\left.\mathbb{R}^{2}, Y_{e} \times \mathbb{R}\right)$, and if $F$ is even, then $\mathcal{H}$ also provides a map $\mathcal{H} \in C^{\omega}\left(X_{e o} \times \mathbb{R}^{2}, Y_{e o} \times \mathbb{R}\right)$.

3.2. Simple bifurcation from $p=2$. The following theorem shows that the zero-Hamiltonian problem associated with (1.1) has a simple bifurcation point to a locally unique and smooth branch of solutions from the point $p=2$.

THEOREM 3.1. Suppose that assumption $(\mathbf{F})$ holds. Then there is an interval $I \subset \mathbb{R}$ and a unique analytic branch $\beta \mapsto(u(\beta), p(\beta)) \in X_{e} \times \mathbb{R}$ defined on $I$ of nontrivial even periodic solutions of (1.1) with zero Hamiltonian and period $T(\beta)$. Moreover, $u(\beta) \neq 0$ if $\beta \neq 0$,

$$
T(0)=2 \pi, u(0)=0, p(0)=2 \text {, and }\|u(\beta)(t)-\beta \cos (t)\|_{C^{4}}=O\left(\beta^{2}\right)
$$

as $\beta \rightarrow 0$. If $F$ is even, then the function $t \mapsto u(\beta)(t)$ is an element of $X_{\text {eo }}$ for all $\beta \in I$.

Proof. To prove this result we apply Theorem 2.2 to $\mathcal{H}(u, p, \mu)=0$ with $X=X_{e}$ and $Y=Y_{e}$. Let $L(p, \mu)[a] \equiv d_{u} M(0, p, \mu)[a]=\mu^{4} a^{\prime \prime \prime \prime}+p \mu^{2} a^{\prime \prime}+a$, and note that the bilinear form $\psi\left(d_{u u}^{2} g(0, p, \mu)[a, b]\right)$ from Theorem 2.2 is given by

$$
B(p, \mu)[a, b]=\delta\left(\mu^{4}\left(a^{\prime} b^{\prime \prime \prime}+a^{\prime} b^{\prime \prime \prime}-a^{\prime \prime} b^{\prime \prime}\right)+p \mu^{2} a^{\prime} b^{\prime}+a b\right) .
$$

In order to verify the hypotheses of Theorem 2.2 let us seek a nonzero solution $a \in X_{e}$ to $L(p, \mu) a=0$, that is,

$$
\mu^{4} a^{\prime \prime \prime \prime}+p \mu^{2} a^{\prime \prime}+a=0, \quad \delta\left(\mu^{4}\left(2 a^{\prime} a^{\prime \prime \prime}-\left(a^{\prime \prime}\right)^{2}\right)+p \mu^{2}\left(a^{\prime}\right)^{2}+a^{2}\right)=0 .
$$

Since $a$ is even and of period $2 \pi$, we seek solutions of the form $a(t)=\cos (m t)$, where $m$ is an integer. This provides the equations $\alpha^{4}-p \alpha^{2}+1=0,-\alpha^{4}+1=0$, where $\alpha=\mu m$, whence $\alpha^{2}=1$, so that $p=2$ and $\mu=1 / m$. Seeking the solution of minimal period, we may set $m=1$ and thus define $k(t) \equiv \cos (t)$ and record the fact that $\operatorname{ker}(L(2,1))=\langle k\rangle$. Let us also define $K \equiv k$ for the purposes of Theorem 2.2 and note for the moment that $k$ is an even-odd function.

We now form the decompositions $X_{e}=\langle k\rangle \oplus\langle k\rangle^{\perp}$ and $Y_{e}=\langle k\rangle \oplus\langle k\rangle^{\perp}$, so that $U=\langle k\rangle^{\perp} \subset X_{e}$ and $V=\langle k\rangle^{\perp} \subset Y_{e}$ in accordance with Theorem 2.2, and define the projection $Q: L^{2}(0,2 \pi) \rightarrow \mathbb{R}$ by

$$
(Q u)(t)=\frac{1}{\pi} \int_{0}^{2 \pi} u(t) k(t) d x, \quad u \in L^{2}(0,2 \pi),
$$

and let $P=I-k \cdot Q$. Now, $\psi\left(d_{\text {uup }}^{3} g(0, p, \mu)[a, b, 1]\right)=B_{p}(p, \mu)[a, b]=\delta\left(\mu^{2} a^{\prime} b^{\prime}\right)$, $d_{u u \mu}^{3} g(0, p, \mu)[a, b]=\delta\left(4 \mu^{3}\left(a^{\prime} b^{\prime \prime \prime}+a^{\prime} b^{\prime \prime \prime}-a^{\prime \prime} b^{\prime \prime}\right)+2 p \mu a^{\prime} b^{\prime}\right)$, and $d_{u \mu}^{2} M(0, p, \mu)[a]=$ 
$L_{\mu}(p, \mu)[a]=4 \mu^{3} a^{\prime \prime \prime \prime}+2 p \mu a^{\prime \prime}$. Finally, $d_{u p}^{2} M(0, p, \mu)[a, 1]=\mu^{2} a^{\prime \prime}$. Thus the remaining hypothesis of Theorem 2.2 is satisfied if the operator matrix $D$ is nonsingular. On inspection of the relevant derivatives we find

$$
D=\left(\begin{array}{crr}
P \circ L(2,1) & 0 & 0 \\
0 & -1 & 0 \\
* & 0 & -4
\end{array}\right) \in B L\left(U \times \mathbb{R}^{2}, V \times \mathbb{R}^{2}\right),
$$

where $P \circ L(2,1): U \rightarrow V$ is an isomorphism and $*$ is irrelevant to the calculation at hand. It follows that $D$ is an isomorphism, and the result follows.

The second part of the theorem is proven in exactly the same way, simply observing the change of space, using $X_{e o}$ rather than $X_{e}$. The uniqueness of the bifurcating branch in both $X_{e}$ and $X_{e o}$, and the fact that $X_{e o} \subset X_{e}$, implies that $u(\beta) \in X_{e o}$ if $F$ is even.

Remark 1. In order to demonstrate that the application of Theorem 2.2 is not limited to fourth-order problems, we present the following example. In [33] the authors study the problem of finding periodic solutions for sixth-order problems using a variational approach, of which

$$
u^{v i}+5 u^{i v}+p u^{\prime \prime}+u-u^{3}=0
$$

is an example (see also [10]). Equation (3.3) has Hamiltonian

$$
H \equiv \frac{1}{2}\left(u^{\prime \prime \prime}\right)^{2}+u^{v} u^{\prime}-u^{i v} u^{\prime \prime}+5\left(u^{\prime} u^{\prime \prime \prime}-\frac{1}{2}\left(u^{\prime \prime}\right)^{2}\right)+\frac{p}{2}\left(u^{\prime}\right)^{2}+\frac{1}{2} u^{2}-\frac{1}{4} u^{4} .
$$

TheOREM 3.2. The points $(u, p)=\left(0,4 \frac{1}{4}\right)$ and $(0,1+4 \sqrt{2})$ are simple bifurcation points to even periodic solutions of (3.3) with zero Hamiltonian and with period near $2 \pi \sqrt{2}$ and $2 \pi / \sqrt{1+\sqrt{2}}$, respectively.

The proof of Theorem 3.2 is very similar to that of Theorem 3.1, and so we omit it; note that the existence of a locally two-dimensional manifold of positive and negative Hamiltonian solutions also follows from Theorem 2.3.

3.3. Double bifurcations from $p>2$. The following result shows that the interval $[2, \infty)$ contains a dense set of bifurcation points for (3.1)-(3.2).

TheOREm 3.3. Suppose that assumption $(\mathbf{F})$ holds. Then to each $n, m \in \mathbb{N}$ such that $n \geq m+1$ and $\operatorname{gcd}(n, m)=1$ there is an interval $I \subset \mathbb{R}$ and exactly two analytic branches $\beta \mapsto\left(u_{ \pm}(\beta), p_{ \pm}(\beta)\right) \in X_{e} \times \mathbb{R}$ defined on I of even periodic solutions of (1.1) with zero Hamiltonian and period $T_{ \pm}(\beta)$. Moreover, $u_{ \pm}(\beta) \neq 0$ for $\beta \neq 0$,

$$
T_{ \pm}(0)=2 \pi \sqrt{n m}, \quad u_{ \pm}(0)=0, \quad p_{ \pm}(0)=\frac{n}{m}+\frac{m}{n},
$$

and

$$
\left\|u_{ \pm}(\beta)(t)-\beta(m \cos (n t) \pm n \cos (m t))\right\|_{C^{4}}=O\left(\beta^{2}\right)
$$

as $\beta \rightarrow 0$.

Proof. Let us apply Theorem 2.4 to $\mathcal{H}(u, p, \mu)=0$; to identify the functions $k_{1}$ and $k_{2}$ from Theorem 2.4, we consider the linearized problem

$$
L(p, \mu)[a] \equiv \mu^{4} a^{\prime \prime \prime \prime}+p \mu^{2} a^{\prime \prime}+a=0
$$


of (3.1) with $a \in X_{e}$. This linear equation admits an even $2 \pi$-periodic solution of the form $a(t)=\lambda_{1} \cos (m t)+\lambda_{2} \cos (n t)$ with integer $m$ and $n$, provided that $\alpha_{1}=(\mu m)^{2}$ and $\alpha_{2}=(\mu n)^{2}$ both satisfy the equation $\alpha^{2}-p \alpha+1=0$. From this we obtain $\alpha_{1} \alpha_{2}=1$, so that $\mu=\mu_{n, m} \equiv \frac{1}{\sqrt{n m}}$ and $p=\alpha_{1}+\alpha_{2}$, whence $p=p_{n, m} \equiv \frac{n}{m}+\frac{m}{n}$.

Hence we define $k_{1}(t) \equiv \cos (n t), k_{2}(t) \equiv \cos (m t)$, and $u_{1} \equiv k_{1}, u_{2} \equiv k_{2}$. Moreover, using $\psi\left(d_{u u}^{2} g\left(0, p_{n, m}, \mu_{n, m}\right)[a, b]\right)=\delta\left(-\mu_{n, m}^{4} a^{\prime \prime} b^{\prime \prime}+a b\right)$, we have

$$
A=\frac{\left(n^{2}-m^{2}\right)}{n^{2}}, \quad B=0, \quad \text { and } C=-A,
$$

so that $\alpha_{ \pm}= \pm \frac{n}{m}$ in the notation of Theorem 2.4. With $Q_{i}(v)=\frac{1}{\pi} \int_{0}^{2 \pi} v(t) u_{i}(t) d t$ for $i=1,2$, we find $d_{u p}^{2} M\left(0, p_{n, m}, \mu_{n, m}\right)[a, 1]=\mu_{n, m}^{2} a^{\prime \prime}$ and $d_{u \mu}^{2} M\left(0, p_{n, m}, \mu_{n, m}\right)[a, 1]=$ $4 \mu_{n, m}^{3} a^{\prime \prime \prime \prime \prime}+2 p_{n, m} \mu_{n, m} a^{\prime \prime}$. We then evaluate the determinant from Theorem 2.4, which is $-4 \mu_{n, m}^{5} \alpha_{ \pm} n^{2} m^{2}\left(m^{2}-n^{2}\right)$, and the result now follows since this is nonzero.

If $F$ is even, then all the bifurcations which occur for $p \geq 2$ are pitchforks because $u$ is then a solution of (3.1)-(3.2) if and only if $-u$ is. The uniqueness properties of Theorems 2.2 and 2.4 and symmetry then imply that the parameterization of the solution branch satisfies $-u(\beta)=u(-\beta)$. From this we infer that the bifurcation diagram of $\|u(\beta)\|$ (with any suitable norm) plotted against $p(\beta)$ has a tongue-like appearance because of the density of the union of $p_{n, m}$ in $[2, \infty)$.

Remark 2. Theorem 3.3 was essentially known some time ago and can be found in an unpublished letter by J. F. Toland (1992), as referred to in [9, equation (5.1), p. 2486] for the case $F_{u}(u)=u-u^{2}$. This letter was communicated to the present authors by A. R. Champneys, and we express our gratitude for his help in this matter. A singularly perturbed version of (1.1) for this choice of nonlinearity was studied in [15] and more recently in [16], where the authors consider both homoclinic and periodic solutions, although the latter are not of zero Hamiltonian; see also [23].

As an aside, consider the equation

$$
\frac{1}{12} v^{i v}+v^{\prime \prime}+p v+v^{3}+\frac{3}{4} v\left(v v^{\prime \prime}+\left(v^{\prime}\right)^{2}\right),
$$

taken from [21], with first integral

$$
H \equiv \frac{1}{12}\left(v^{\prime \prime \prime} v^{\prime}-\frac{1}{2}\left(v^{\prime \prime}\right)^{2}\right)+\frac{1}{2}\left(v^{\prime}\right)^{2}+\frac{p}{2} v^{2}+\frac{v^{4}}{4}+\frac{3}{8}\left(v^{\prime}\right)^{2} v^{2} .
$$

Note that a parameter $\lambda^{2}$ appearing in [21] has been replaced here by $p$. This is not in the class of Hamiltonian systems given by (1.1), but Theorems 2.2 and 2.4 are still applicable.

THEOREM 3.4. The point $p=3$ is a simple bifurcation point to even periodic solutions of (3.5) with zero first integral and with period near $\pi \sqrt{2 / 3}$. For each $n, m \in$ $\mathbb{N}$ such that $n>m$ and $\operatorname{gcd}(n, m)=1$, the point $p_{n, m}=12\left(\frac{n}{m}+\frac{m}{n}\right)^{-2}$ is a double bifurcation point to such solutions with period near $\pi \sqrt{\left(n^{2}+m^{2}\right) / 3}$.

The proof of Theorem 3.4 is an application of Theorem 2.4, which is entirely analogous to the proof of Theorem 3.3, so we omit the details.

3.4. Odd solutions for even $\boldsymbol{F}$. Now let us suppose that $F$ is an even function. If we define the spaces of odd functions, $X_{o}=\left\{u \in C_{2 \pi}^{4}: u(t)=-u(-t)\right\}$ and $Y_{o}=$ $\left\{u \in C_{2 \pi}^{0}: u(t)=-u(-t)\right\}$, then $\mathcal{H}$ provides a map $\mathcal{H} \in C^{\omega}\left(X_{o} \times \mathbb{R}^{2}, Y_{o} \times \mathbb{R}\right)$. This means that one can obtain odd zero-Hamiltonian solutions of (1.1) in a manner entirely 
analogous to the way we found the even solutions. For this reason we give the following theorem without proof.

THEOREM 3.5. If assumption (F) holds and $F$ is even, then to each $n, m \in \mathbb{N}$ such that $n \geq m+1$ and $\operatorname{gcd}(n, m)=1$ there is an interval $I \subset \mathbb{R}$ and exactly two analytic branches $\beta \mapsto\left(u_{ \pm}(\beta), p_{ \pm}(\beta)\right) \in X_{e} \times \mathbb{R}$ defined on $I$ of odd periodic solutions of (1.1) with zero Hamiltonian and period $T_{ \pm}(\beta)=2 \pi / \mu_{ \pm}(\beta)$. Moreover, $u_{p} m(\beta) \neq 0$ for $\beta \neq 0$,

$$
T_{ \pm}(0)=2 \pi \sqrt{n m}, \quad u_{ \pm}(0)=0, \quad p_{ \pm}(0)=\frac{n}{m}+\frac{m}{n}
$$

and

$$
\left\|u_{ \pm}(\beta)(t)-\beta(m \sin (n t) \pm n \sin (m t))\right\|_{C^{4}}=O\left(\beta^{2}\right)
$$

as $\beta \rightarrow 0$.

Of course, there is little point in formulating a version of Theorem 3.1 in this context, since that theorem already tells us that a branch of odd solutions of (1.1) can be found by shifting time.

It is possible to formulate an extension of the results proven in this section by considering a smooth one-parameter family of reversible vector fields on $\mathbb{R}^{n}$ which possesses a trivial branch of equilibrium solutions and a first integral. One could use Theorems 2.2 and 2.4 to formulate sufficient conditions for the bifurcation of zeroenergy symmetric periodic solutions. However, for brevity we have not done this, and we restrict our attention to the properties of fourth-order systems.

3.5. Disjointness properties of solution sets. Motivated by Theorems 3.1 and 3.3, we define the following nonempty sets, assuming $(\mathbf{F})$ to be true. Let

$$
\Sigma \equiv\left\{(u, p, \mu) \in X_{e} \times \mathbb{R} \times \mathbb{R}: \mathcal{H}(u, p, \mu)=0, u \neq 0, \mu>0\right\},
$$

and let $\bar{\Sigma}$ denote the closure of $\Sigma$ in $X_{e} \times \mathbb{R}^{2}$. For any pair $(n, m) \in \mathbb{N} \times \mathbb{N}$ such that $\operatorname{gcd}(n, m)=1$, let $C(n, m)$ be the maximal connected subset of $\bar{\Sigma}$ which contains the point $(u, p, \mu)=\left(0, p_{n, m}, \mu_{n, m}\right)$, and define the functional $\nu: \Sigma \rightarrow(2, \infty)$ by

$$
\nu(u, p, \mu)=\|u\|_{C^{4}}+|p|+|\mu|+\frac{1}{|\mu|} .
$$

Also, let

$$
\Sigma_{+}=\{(u, p, \mu) \in \Sigma: p>0\},
$$

$\bar{\Sigma}_{+}$be the closure of $\Sigma_{+}$, and $C_{+}(n, m)$ be the maximal connected subset of $C(n, m) \cap$ $\bar{\Sigma}_{+}$which contains the point $(u, p, \mu)=\left(0, p_{n, m}, \mu_{n, m}\right)$.

We continue with a simple lemma which is used in the subsequent analysis. Throughout this section, \# is used to represent the cardinality of a set, and we introduce a potential $V\left(u, u^{\prime \prime}\right)$ by writing (3.2) as

$$
-\mu^{2} u^{\prime}\left(\mu^{2} u^{\prime \prime \prime}+\frac{p}{2} u^{\prime}\right)=V\left(u, u^{\prime \prime}\right) \equiv-\frac{1}{2} \mu^{4} u^{\prime \prime 2}+F(u) .
$$

Lemma 3.1. Suppose that $n, m \geq 1$ are distinct integers; then $\#\{t \in[0, \pi]$ : $n \cos (n t) \pm m \cos (m t)=0\}=\max (n, m)$ and $\#\{t \in[0, \pi]: m \cos (n t) \pm n \cos (m t)=$ $0\}=\min (n, m)$. 
The following two theorems provide bifurcation invariants that are invaluable to the study of the global nature of $\Sigma$.

THEOREM 3.6. If assumption $(\mathbf{F})$ holds and $F(u)>0$ for $u \neq 0$, then the mapping

$$
\iota_{1}: \Sigma \rightarrow \mathbb{N} ;(u, p, \mu) \mapsto \#\{t \in[0, \pi]: u(t)=0\}
$$

is continuous and satisfies $\iota_{1}\left(C(n, m) \backslash\left\{\left(0, p_{n, m}, \mu_{n, m}\right)\right\}\right) \equiv \min (n, m)$.

We postpone the proof of this theorem until after the following preliminary lemma.

LEMMA 3.2. Let $\left(u_{k}, p_{k}, \mu_{k}\right) \subset \Sigma$ be a sequence with $\left(u_{k}, p_{k}, \mu_{k}\right) \rightarrow(u, p, \mu) \in \Sigma$, and suppose that there is a pair of sequences $\left(t_{k}^{1}\right),\left(t_{k}^{2}\right) \subset[0,2 \pi]$ such that $\left|t_{k}^{1}-t_{k}^{2}\right| \rightarrow 0$ and $u_{k}^{\prime}\left(t_{k}^{1,2}\right)=0$. Then $u_{k}\left(t_{k}^{1}\right) u_{k}\left(t_{k}^{2}\right)>0$ for sufficiently large $k$.

Proof. For definiteness we assume that $t_{k}^{1}<t_{k}^{2}$ and, seeking a contradiction, we also assume that $u_{k}\left(t_{k}^{1}\right) u_{k}\left(t_{k}^{2}\right) \leq 0$; we initially also assume that $u_{k}\left(t_{k}^{1}\right)<0<u_{k}\left(t_{k}^{2}\right)$. By the mean-value theorem there is a $T_{k} \in\left(t_{k}^{1}, t_{k}^{2}\right)$ such that $u_{k}^{\prime \prime}\left(T_{k}\right)=0$, and taking the limit $k \rightarrow \infty$ gives the existence of a $T$ such that $u^{\prime}(T)=u^{\prime \prime}(T)=0$. Using (3.2) yields $F(u(T))=0$ and therefore $u(T)=0$, and since $u$ is not identically zero, it follows that $u^{\prime \prime \prime}(T) \neq 0$.

We now assume without the loss of any generality that $\left\|u_{k}\right\|_{C^{4}} \leq\|u\|_{C^{4}}+1 \equiv B$ and that $\frac{1}{2} \mu \leq \mu_{k} \leq \frac{3}{2} \mu$. By the smoothness of $F$ and since $F(0)=F_{u}(0)=0$, we may assume that there is a $C$ such that

$$
|F(w)| \leq \frac{C^{2}}{2}|w|^{2} \quad \text { if }|w| \leq B
$$

Since $u_{k}^{\prime}\left(t_{k}^{1,2}\right)=0$, we have $V\left(u_{k}, u_{k}^{\prime \prime}\right)=-\frac{1}{2} \mu_{k}^{4}\left(u_{k}^{\prime \prime}\right)^{2}+F\left(u_{k}\right)=0$ at $t_{k}^{1,2}$, and therefore

$$
\left|u_{k}^{\prime \prime}\right| \leq \frac{C}{\mu_{k}^{2}}\left|u_{k}\right| \leq \frac{4 C}{\mu^{2}}\left|u_{k}\right| \quad \text { at } t_{k}^{1,2} .
$$

If we define the function $v(t)=V\left(u_{k}(t), u_{k}^{\prime \prime}(t)\right)$, then the mean-value theorem gives the existence of a $\tau_{k} \in\left(t_{k}^{1}, t_{k}^{2}\right)$ with $v^{\prime}\left(\tau_{k}\right)=0$. From (3.10), the orbit of $u_{k}$ is a smooth curve in the $u, u^{\prime \prime}$-plane that connects $\left(u_{k}, u_{k}^{\prime \prime}\right)\left(t_{k}^{1}\right)$ (in the left half-plane) to $\left(u_{k}, u_{k}^{\prime \prime}\right)\left(t^{2}\right)$ (in the right half-plane) with end-points in the region $\left\{\left(u, u^{\prime \prime}\right):\left|u^{\prime \prime}\right| \leq\right.$ $\left.\frac{4 C}{\mu^{2}}|u|\right\}$. By considering the tangent vector $\left(u^{\prime}, u^{\prime \prime \prime}\right)$ of this planar curve, it follows that there is a $\hat{\tau}_{k} \in\left(t_{k}^{1}, t_{k}^{2}\right)$ such that

$$
\left|u_{k}^{\prime \prime \prime}\left(\hat{\tau}_{k}\right)\right| \leq \frac{4 C}{\mu^{2}}\left|u_{k}^{\prime}\left(\hat{\tau}_{k}\right)\right| .
$$

When we take the limit $k \rightarrow \infty$, it follows from the estimate

$$
\sup _{\left(t_{k}^{1}, t_{k}^{2}\right)}\left|u_{k}^{\prime}\right| \leq\left|t_{k}^{2}-t_{k}^{1}\right| \sup _{\left(t_{k}^{1}, t_{k}^{2}\right)}\left|u_{k}^{\prime \prime}\right|
$$

that $u_{k}^{\prime \prime \prime}\left(\hat{\tau}_{k}\right) \rightarrow 0$. We therefore find that the limiting solution $u$ satisfies $u^{\prime \prime \prime}(T)=0$, which is a contradiction.

If the signs of $u_{k}$ at $t_{k}^{1,2}$ are inverted, so that $u_{k}\left(t_{k}^{2}\right)<0<u_{k}\left(t_{k}^{1}\right)$, then the argument given above holds unchanged. If exactly one of the two values $u_{k}\left(t_{k}^{1,2}\right)$ is zero for all $k$, then the argument holds in a similar way: in this case the curve in the $u, u^{\prime \prime}$-plane connects the origin to the other point. The existence of $\hat{\tau}_{k}$ satisfying (3.11) 
follows as before. If both of the values of $u_{k}$ are zero, then the curve is closed, and again a value of $\hat{\tau}_{k}$ can be found satisfying (3.11). This concludes the proof of the lemma.

Proof of Theorem 3.6. Suppose that $(u, p, \mu) \in \Sigma$ and that $\left(u_{k}, p_{k}, \mu_{k}\right) \subset \Sigma$ satisfies $\left(u_{k}, p_{k}, \mu_{k}\right) \rightarrow(u, p, \mu)$ in $C^{4} \times \mathbb{R}^{2}$.

We first note that if four or more zeros of $u_{k}$ collide (counted according to algebraic multiplicity), say $0 \leq t_{k}^{1} \leq t_{k}^{2} \leq t_{k}^{3} \leq t_{k}^{4} \leq 2 \pi$ are all zeros of $u_{k}$ that converge to $T$, then from the mean-value theorem we have $u(T)=u^{\prime}(T)=u^{\prime \prime}(T)=u^{\prime \prime \prime}(T)=0$, contradicting the assumption that $u \neq 0$.

On the other hand, if two or more zeros collide, then from the mean-value theorem again there is a $T$ such that $u^{\prime}(T)=0$ and therefore $u^{\prime \prime}(T)=0$ by (3.2). In order to avoid the same contradiction as above, necessarily $u^{\prime \prime \prime}(T) \neq 0$. This implies that the zero of $u$ at $t=T$ is topologically transverse, which rules out the possibility that two transverse zeros coalesce.

We therefore are left with two cases: either three simple zeros collide or two zeros collide of which one is a double zero. In the first case, three simple zeros, there exist $t_{k}^{1,2}$ such that $u_{k}^{\prime}\left(t_{k}^{1,2}\right)=0$ and $t_{k}^{1,2} \rightarrow T$ as $k \rightarrow \infty$, and since the zeros are transverse, we can assume that $u_{k}$ has opposite signs at $t_{k}^{1}$ and $t_{k}^{2}$. The conclusions of Lemma 3.2 show that this situation leads to a contradiction. In the second case we choose $t_{k}^{1}$ to be the nonsimple zero, and $\tau_{k} \in\left(t_{k}^{1}, t_{k}^{2}\right)$ to be an intermediate point such that $u^{\prime}\left(\tau_{k}\right)=0$. Again an application of Lemma 3.2 leads to a contradiction, and therefore the number of zeros of $u_{k}$ eventually equals that of $u$. This shows that $\iota_{1}$ is continuous on $\Sigma$ and therefore constant on connected components of $\Sigma$.

In order to evaluate $\iota_{1}(u, p, \mu)$ for $(u, p, \mu) \in C(n, m)$ with $u \neq 0$ we use the representation of $C(n, m)$ at bifurcation given in Theorem 3.3. From Lemma 3.1 there results

$$
\left\|u_{ \pm}(\beta)(t)-\beta(m \cos (n t) \pm n \cos (m t))\right\|_{C^{4}}=O\left(\beta^{2}\right),
$$

and hence $\#\left\{t \in[0, \pi]: u_{ \pm}(\beta)(t)=0\right\}=\min (n, m)$ follows for sufficiently small and nonzero $\beta$, and the theorem is proven.

THEOREM 3.7. If assumption $(\mathbf{F})$ holds and $F(u)>0$ for $u \neq 0$, then the mapping

$$
\iota_{2}: \Sigma_{+} \rightarrow \mathbb{N} ;(u, p, \mu) \mapsto \#\left\{t \in[0, \pi]: u^{\prime \prime}(t)=0\right\}
$$

is continuous.

Proof. Let $(u, p, \mu) \in \Sigma_{+}$, and suppose that there is a $T \in[0,2 \pi]$ such that

$$
u^{\prime \prime}(T)=u^{\prime \prime \prime}(T)=0 .
$$

The zero-Hamiltonian condition (3.2) then gives

$$
\frac{1}{2} p \mu^{2} u^{\prime}(T)^{2}+F(u(T))=0,
$$

and the hypotheses on $F$ ensure that $u^{\prime}(T)=0$ and $u(T)=0$. It follows that $u=0$, which contradicts the definition of $\Sigma_{+}$, and this contradiction implies that the zeros of $u^{\prime \prime}$ are transverse. Consequently, if $\left(u_{n}, p_{n}, \mu_{n}\right) \subset \Sigma_{+}$is a sequence such that $\left(u_{n}, p_{n}, \mu_{n}\right) \rightarrow(u, p, \mu)$ in $\Sigma_{+}$, then $u_{n}^{\prime \prime} \rightarrow u^{\prime \prime}$ in the $C^{1}$ topology. Hence $u_{n}^{\prime \prime}$ has the same number of zeros as $u^{\prime \prime}$ for all sufficiently large $n$, which shows that $\iota_{2}$ is continuous as claimed. 
Theorem 3.7 immediately implies that $\iota_{2}$ is constant on connected components of $\Sigma_{+}$, and from this observation we deduce the following.

Corollary 3.1. Suppose that $(\mathbf{F})$ holds and $u F_{u}(u) \geq 0$ for all $u \in \mathbb{R}$; then $\Sigma_{+}=\Sigma$, and as a consequence, $C_{+}(n, m)=C(n, m)$. Moreover,

$$
\iota_{2}\left(C(n, m) \backslash\left\{\left(0, p_{n, m}, \mu_{n, m}\right)\right\}\right)=\max (n, m),
$$

so that $C(n, m) \cap C\left(n^{\prime}, m^{\prime}\right)$ is empty unless $(n, m)=\left(n^{\prime}, m^{\prime}\right)$.

Proof. Multiplying (3.1) by $u$ and integrating gives

$$
p \mu^{2} \int_{0}^{2 \pi}\left(u^{\prime}\right)^{2} d t=\int_{0}^{2 \pi} \mu^{4}\left(u^{\prime \prime}\right)^{2}+u F_{u}(u) d t \geq \int_{0}^{2 \pi} \mu^{4}\left(u^{\prime \prime}\right)^{2} d t \geq 0 .
$$

Hence if there is a solution of (3.1)-(3.2) with $p=0$ and $\mu>0$, it follows that $u^{\prime \prime} \equiv 0$, so $u(t)=A t+B$ for constants $A$ and $B$. As $u$ is periodic, $A=0$, and as $u$ must have zero Hamiltonian, $F(B)=0$ is also true. The hypotheses ensure that $F(u)=0$ only when $u=0$ so that $B=0$; hence $u(t) \equiv 0$ and so $\Sigma=\Sigma_{+}$, from which $C(n, m)=C_{+}(n, m)$ by definition.

Since $\iota_{2}: \Sigma \rightarrow \mathbb{N}$ is continuous by Theorem 3.7, the set $\mathcal{C}$ defined by $\mathcal{C} \equiv$ $C(n, m) \backslash\left\{\left(0, p_{n, m}, \mu_{n, m}\right)\right\}$ is a connected subset of $\Sigma$, because the intersection of $C(n, m)$ with some small ball, $C(n, m) \cap B_{\delta}\left(0, p_{n, m}, \mu_{n, m}\right)$, is path-connected for all sufficiently small $\delta>0$. Hence $\iota_{2}$ is constant on $\mathcal{C}$. In order to evaluate $\iota_{2}(u, p, \mu)$ with $(u, p, \mu) \in \mathcal{C}$ we use the representation of $C_{+}(n, m)$ at bifurcation from the trivial solution described in Theorem 3.3 and then apply Lemma 3.1. From Theorem 3.3 we have

$$
\left\|u_{ \pm}^{\prime \prime}(\beta)(t)+\beta m n(n \cos (n t) \pm m \cos (m t))\right\|_{C^{2}}=O\left(\beta^{2}\right),
$$

whence $\#\left\{t \in[0, \pi]: u_{ \pm}^{\prime \prime}(\beta)(t)=0\right\}=\max (n, m)$ follows for sufficiently small and nonzero $\beta$.

Finally, as $(\mathbf{F})$ ensures that $F$ is positive in a punctured neighborhood of zero, the condition $u F_{u}(u) \geq 0$ then ensures that $F(u)>0$ if $u \neq 0$. Applying Theorem 3.6, we obtain $\iota_{j}(C(n, m))=\iota_{j}\left(C\left(n^{\prime}, m^{\prime}\right)\right)$ for $j=1,2$, and the last part of the corollary follows.

Finally, we have the following theorem, which applies to path-connected subsets of $\Sigma$, although it provides no information regarding the behavior of connected subsets of $\Sigma$ which are not path-connected.

ThEOREM 3.8. Suppose that $F(u)>0$ for $u \neq 0$, and let $\left(u_{s}, p_{s}\right)$ be a continuous path of solutions of (1.1), where each $u_{s}$ is defined on a sufficiently large subset of $\mathbb{R}$ and the path does not contain the equilibrium solution. If two zeros of $u_{s}^{\prime}$ collide, then multiplicity is preserved in and through the collision.

Proof. We may assume that all collisions occur at $s=0$ and $t=0$. Note that if all $u_{s}$ are defined on a common interval $I \subset \mathbb{R}$, then by standard elliptic estimates the solution curve is bounded in $C^{k}\left(I^{\prime}\right)$ for any $k \in \mathbb{N}$ and any compact interval $I^{\prime} \subset I$. Since $F$ is smooth, we can bound $F$ by

$$
|F(w)| \leq \frac{C^{2} w^{2}}{2}
$$

for, say, $|w| \leq 1$.

Note that $u^{\prime}(0)=u^{\prime \prime}(0)=0$ at $s=0$, and therefore by $(1.2), F(u(0))=0$, which implies $u(0)=0$. The nonconstancy hypothesis on each $u_{s}$ implies that $u^{\prime \prime \prime}(0) \neq 0$. 


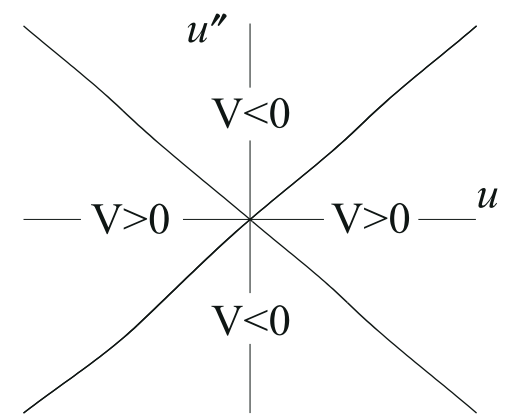

FIG. 3.1. The configuration space $u, u^{\prime \prime}$ is partitioned according to the sign of $V$.

This proves that multiplicity is conserved in the collision. Note that the zero at $(t, s)=(0,0)$ is necessarily transverse.

To show that any subsequent perturbation preserves the multiplicity, we use some ideas from the analysis of the configuration space $\left(u, u^{\prime \prime}\right)$ from [34, 17]; [29] contains a simplified description that is sufficient for our purposes. The structure of the configuration space and the set $\{V=0\}$ is shown in Figure 3.1. Near the origin in this plane the set $\{V=0\}$ consists of two curves that intersect in the origin. Near the origin the direction of these curves is bounded from above by $2 C$, where $C$ is the constant in (3.12).

At $s=0$, we have $u(0)=u^{\prime}(0)=u^{\prime \prime}(0), u^{\prime \prime \prime}(0) \neq 0$, and therefore the orbit near $t=0$ is represented in the $u, u^{\prime \prime}$-plane by a curve that remains inside the set $\{V \leq 0\}$ and intersects $\{V=0\}$. We can choose appropriate translations of $u_{s}$, and small $\bar{t}, \bar{s}>0$, such that we have the following:

1. $u_{s}(t)$ is defined for $(t, s) \in Q \equiv(-\bar{t}, \bar{t}) \times(-\bar{s}, \bar{s})$.

2. $u_{s}$ depends smoothly on $s$ in $C^{4}(-\bar{t}, \bar{t})$.

3. $u_{s}^{\prime \prime \prime}(t) \geq 0$ on $Q$ (if not, then reverse time).

4. For each $s \in(-\bar{s}, \bar{s})$ we have $\pm u_{s}^{\prime \prime}( \pm \bar{t})<0$, and $V\left(u_{s}( \pm \bar{t}), u_{s}^{\prime \prime}( \pm \bar{t})\right)<0$. This implies that no intersections of the solutions with $\{V=0\}$ appear or disappear through the boundary $\pm \bar{t}$.

5. $u_{s}^{\prime \prime \prime} / u_{s}^{\prime} \geq 4 C$ on $Q$, where $C$ is the constant in (3.12).

We write $\gamma_{s} \equiv\left\{\left(u_{s}(t), u_{s}^{\prime \prime}(t)\right):-\bar{t}<t<\bar{t}\right\}$.

We now consider the alternatives for perturbation away from $s=0$. First assume that $\bar{s}$ can be chosen such that $\gamma_{s} \cap\{V=0\}$ has only one intersection for $0<s<\bar{s}$; let this intersection be at $0<t_{s}<\bar{s}$. The lower bound on the angle of the curve $\gamma_{s}$, given by condition 5 above, implies that $\gamma_{s}$ intersects $\{V=0\}$ only at the origin in the $u, u^{\prime \prime}$ plane. Since $u_{0}^{\prime}(0)=0$ and $u_{0}^{\prime \prime \prime}(0) \neq 0$, the smooth dependence of $u_{s}$ on $s$ implies that $u_{s}^{\prime \prime \prime}\left(t_{s}\right) \neq 0$ for $s$ close to zero; therefore the requirement $u^{\prime}\left(t_{s}\right)\left(u_{s}^{\prime \prime \prime}\left(t_{s}\right)+p u_{s}^{\prime}\left(t_{s}\right) / 2\right)=0$ forces $u_{s}^{\prime}\left(t_{s}\right)=0$. Combining this with $u_{s}\left(t_{s}\right)=0$ and taking the limit yields that the zero of $u^{\prime}$ at $s=0$ is a double zero.

To cover the alternative case we assume that $\gamma_{s} \cap\{V=0\}$ has at least two intersections for a sequence $0<s_{n}<\bar{s}, s_{n} \downarrow 0$, at the points $0<t_{n}<\tau_{n}<\bar{t}$. We have $\lim _{n \rightarrow \infty} t_{n}=\lim _{n \rightarrow \infty} \tau_{n}=0$. With an argument similar to the one above, it follows that $u_{s_{n}}^{\prime}\left(t_{n}\right)=u_{s_{n}}^{\prime}\left(\tau_{n}\right)=0$, and therefore the zero at $s=0$ is of second order.

At any point where the orbit intersects $\{V=0\}$, either $u^{\prime}=0$ or $u^{\prime \prime \prime}=-p u^{\prime} / 2$. Since at $s=0$ we have $u^{\prime}=0$ and $u^{\prime \prime \prime} \neq 0$, under perturbation of $s$ we have $u^{\prime}=0$ on the intersection of the orbit with $\{V=0\}$. If we assume that under perturbation 


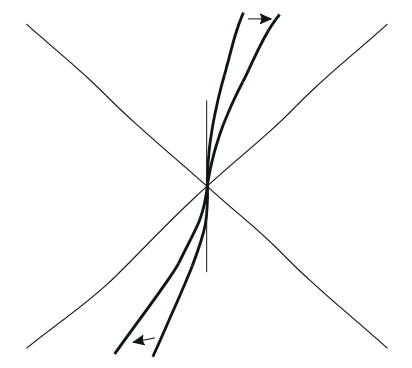

(a)

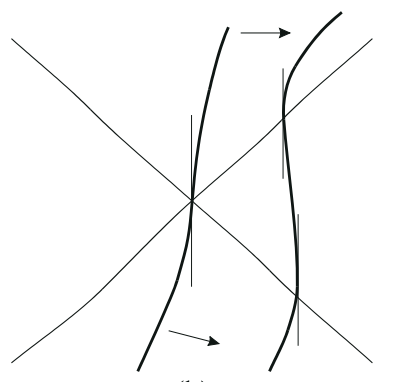

(b)

FIG. 3.2. Two forms of perturbation: (a) if the curve continues to intersect $\{V=0\}$ in the origin, then the tangent remains vertical; (b) a translation, on the other hand, creates new zeros of $u^{\prime}$ and therefore also conserves multiplicity.

there is only one zero of $u^{\prime}$ (locally), then the intersection with $\{V=0\}$ necessarily occurs at the origin in the $u, u^{\prime \prime}$-plane. Therefore the zero remains of multiplicity two.

Note that the only possible scenario is the reduction of multiplicity two to multiplicity zero. Multiplicity zero implies that while $u^{\prime}(0)=0$ at $s=0$, this zero of $u^{\prime}$ disappears under perturbation. An inspection of the $u, u^{\prime \prime}$-plane (Figure 3.2) shows that such a perturbation is possible only if $u^{\prime \prime \prime}+p u^{\prime} / 2$, which is nonzero at $s=0$, jumps to zero for $s \neq 0$. This contradicts the assumption of continuous dependence of the curve of solutions in $C^{k}$ on the parameter $s$.

4. Global bifurcations. Let us now briefly state some results from global realanalytic bifurcation theory for one-parameter problems as developed in [6]. The utility of this theory with respect to (2.1) is the fact that Lemma 2.1 identifies either $p$ or $\mu$ which can be used as the bifurcation parameter. So, supposing that $\mathcal{U} \subset \mathbb{R} \times X$ is a given set and that $F: \mathbb{R} \times X \rightarrow Y$ is a real analytic map, define the set

$$
S=\left\{(\lambda, x) \in \mathcal{U}: F(\lambda, x)=0, d_{x} F(\lambda, x) \in \operatorname{Iso}(X, Y)\right\} .
$$

Throughout this section the space $\mathbb{R} \times X$ is endowed with the norm $|\lambda|+\|x\|_{X}$, and a pair $(\lambda, x) \in S$ is said to be a regular solution of $F(\lambda, x)=0$. While the theory developed in [6] is more powerful than we require, we shall use the following result, which is the statement of Theorem 7.4(iii) of this reference.

THEOREM 4.1. Let $\nu: \mathbb{R} \times X \rightarrow[0, \infty)$ be a given function, and suppose that

(i) $S$ is nonempty and $\mathcal{U} \cap \mathcal{S}$ is open in $\mathcal{S}$, where $\mathcal{S} \equiv\{(\lambda, x) \in \mathbb{R} \times X: F(\lambda, x)=$ $0\}$,

(ii) $d_{x} F(\lambda, x)$ is Fredholm of index zero for all $(\lambda, x) \in \mathcal{U}$,

(iii) subsets of $\mathcal{S}$ on which $\nu$ is bounded have compact closure,

(iv) there are $\delta>0, \lambda_{0} \in \mathbb{R}$, and an analytic function $h: N_{\delta}\left(\lambda_{0}\right) \backslash\left\{\lambda_{0}\right\} \rightarrow S$, where $N_{\delta}\left(\lambda_{0}\right)$ is a half-neighborhood of $\lambda_{0}$, such that $\lim _{\lambda \rightarrow \lambda_{0}} h(\lambda)=0$ but $\left(\lambda_{0}, 0\right) \notin \mathcal{U}$

(v) if $\mathcal{A}_{0}$ is the maximal path-connected subset of $S$ which contains the graph of $h$ and if $\left(\xi_{n}\right) \subset \bar{S} \cap \mathcal{U}$ is any convergent sequence with $\xi_{n} \rightarrow \xi \notin \mathcal{U}$ and $\sup _{n} \nu\left(\xi_{n}\right)<\infty$, then $\xi=\left(\lambda_{0}, 0\right)$ and $\xi_{n} \in \mathcal{A}_{0}$ for all $n$ sufficiently large.

Under conditions (i)-(v) the maximal connected component of $\bar{S} \cap \mathcal{U}$ that contains $\mathcal{A}_{0}$ contains a path-connected subset $\mathcal{P}$ on which $\nu$ is unbounded.

If we recall the definition of the sets $\Sigma$ and $\Sigma_{+}$in (3.7) and (3.9), respectively, then the reasoning in Corollary 3.1 ensures that $\Sigma_{+}=\Sigma$ if $u F_{u}(u) \geq 0$ for all $u \in \mathbb{R}$. 
From this observation we can obtain the following lemma.

Lemma 4.1. Suppose that $(\mathbf{F})$ holds and $u F_{u}(u) \geq 0$ for $u \in \mathbb{R}$; then $\nu(u, p, \mu)$ (defined in (3.8)) is unbounded on a path-connected subset of $C(1,1)$.

Proof. Let us verify the hypotheses of Theorem 4.1 in turn, where $\omega=\mu-1, x=$ $(u, \omega)$ and we write $\lambda$ in place of $p$. To keep the notation consistent with Theorem 4.1, let $F \equiv M \times \psi(g)$ (the symbol $\mathcal{H}$ was used for this previously) and

$$
\mathcal{U} \equiv\left\{(\lambda, x)=(p, u, \omega) \in \mathbb{R} \times X_{e} \times \mathbb{R}: \omega>-1, u \neq 0, \iota_{1}(u)=\iota_{2}(u)=1\right\} .
$$

We remark that $S \subset \Sigma \subset \mathcal{S}, \mathcal{S} \cap \mathcal{U} \subset \Sigma$ and the space $X$ referred to in Theorem 4.1 is $X_{e} \times \mathbb{R}$ and $Y$ is $Y_{e} \times \mathbb{R}$.

(i) It follows from Lemma 2.1 that $S$ is nonempty. (The argument assumes that $d_{u, p}(M \times \psi(g))$ is an isomorphism from Lemma 2.1, for if this is not the case, then we can repeat the argument of this proof using $\mu$ for $\lambda$ rather than $p$.) One can see that the set $\mathcal{U} \cap \mathcal{S}$ is open in $\mathcal{S}$ as follows. Suppose, seeking a contradiction, that $\mathcal{U} \cap \mathcal{S}$ is not open in $\mathcal{S}$, so there is a $\left(\lambda_{0}, x_{0}\right)=\left(p_{0}, u_{0}, \omega_{0}\right) \in \mathcal{S} \cap \mathcal{U}$ and a sequence $\left(\lambda_{n}, x_{n}\right)=$ $\left(p_{n}, u_{n}, \omega_{n}\right) \in \mathcal{S} \backslash \mathcal{U}$ such that $\left(\lambda_{n}, x_{n}\right)=\left(p_{n}, u_{n}, \omega_{n}\right) \rightarrow\left(\lambda_{0}, x_{0}\right)=\left(p_{0}, u_{0}, \omega_{0}\right)$.

Since $u_{0} \neq 0$ and $u_{n} \stackrel{C^{4}}{\rightarrow} u_{0}$, it follows that $u_{n} \neq 0$, and as $\omega_{0}>-1$, then $\omega_{n}>-1$, both for all sufficiently large $n$. Since $\iota_{1}$ and $\iota_{2}$ are continuous functions on $\Sigma$ and $\Sigma_{+}$, respectively, and $\Sigma=\Sigma_{+}$by the hypothesis on $F$, then $\iota_{1}\left(u_{n}\right) \rightarrow \iota_{1}\left(u_{0}\right)=1$ as $n \rightarrow \infty$; but since $\iota_{1}$ is integer-valued, this means that $\iota_{1}\left(u_{n}\right) \equiv 1$ for all $n$ sufficiently large. Similar reasoning applies to $\iota_{2}$. Consequently, $\left(\lambda_{n}, x_{n}\right) \in \mathcal{U}$ for all $n$ sufficiently large, which is the required contradiction.

(ii) The operator $d_{x} F(\lambda, x)$ has the form

$$
\left(\begin{array}{cc}
d_{u} M(u, p, \mu) & 0 \\
0 & 0
\end{array}\right)+K \in B L\left(X_{e} \times \mathbb{R}, Y_{e} \times \mathbb{R}\right)
$$

where $K$ is a continuous operator that has rank at most two and $d_{u} M(u, p, \mu)[h]=$ $\mu^{4} h^{\prime \prime \prime \prime}+p \mu^{2} h^{\prime \prime}+F_{u}(u) h \in B L\left(X_{e}, Y_{e}\right)$. However, the latter is a compact perturbation of the operator

$$
E: h \mapsto \mu^{4} h^{i v}+\theta h, \quad E \in B L\left(X_{e}, Y_{e}\right)
$$

Since $\mu>0$, using a Fourier series argument, one can easily show that there is a $\theta$ such that $E$ is an isomorphism of the given spaces. Consequently $d_{x} F(\lambda, x)$ is a compact perturbation of a Fredholm mapping of index zero, and therefore is itself Fredholm of index zero.

(iii) If $\left(u_{n}, p_{n}, \omega_{n}\right) \subset \mathcal{S}$ is a sequence such that $\omega_{n}=\mu_{n}-1>-1$ and

$$
\nu\left(u_{n}, p_{n}, \mu_{n}\right)=\left\|u_{n}\right\|_{C^{4}}+\left|p_{n}\right|+\left|\omega_{n}+1\right|+\frac{1}{\left|\omega_{n}+1\right|}
$$

is bounded, then there are $p_{0}$ and $\omega_{0}$ such that $\omega_{n} \rightarrow \omega_{0} \geq-1$ and $p_{n} \rightarrow p_{0} \geq 0$. Now $\omega_{0} \neq-1$ by the boundedness of $\nu$, and therefore $u_{n}^{v}=-\left(\omega_{n}+1\right)^{-4}\left(p_{n}\left(\omega_{n}+\right.\right.$ $\left.1)^{-2} u_{n}^{\prime \prime \prime}+F_{u}\left(u_{n}\right) u_{n}^{\prime}\right)$ is also bounded, whence $\left(u_{n}\right)$ converges to some $u_{0} \in C^{4}$ as the embedding $C^{5} \hookrightarrow C^{4}$ is compact, and therefore $\left(p_{n}, u_{n}, \omega_{n}\right)$ converges in $\mathbb{R} \times X_{e} \times \mathbb{R}$.

(iv) This part of the theorem follows from Theorem 2.2 and Lemma 2.1, where the bifurcating branch is represented by an analytic curve of regular solutions.

(v) If a $\nu$-bounded sequence $\left(\xi_{n}\right)=\left(p_{n}, u_{n}, \omega_{n}\right) \subset \bar{S} \cap \mathcal{U}$ satisfies $\xi_{n} \rightarrow \xi=$ $\left(p_{0}, u_{0}, \omega_{0}\right) \notin \mathcal{U}$, then the only viable possibility is that $u_{0}=0$, so that $(p, \mu)=$ 
$\left(p_{0}, \omega_{0}+1\right)$ is a bifurcation point from the trivial solution of (3.1)-(3.2). However, the only point at which such a bifurcation occurs in the set $S$ is at the point $(u, p, \mu)=$ $(0,2,1)$, so that $(u, \omega)=(0,0)$. Hence property (v) is satisfied if $\mathcal{A}_{0}$ is defined to be the maximal path-connected subset of $S$ which contains the graph of the bifurcating branch from Theorem 3.1. In this case let us note that $\lambda_{0}=2$.

This concludes the proof.

Now define the functional on $X_{e} \times \mathbb{R}^{2}$ by

$$
\bar{\nu}(u, p, \mu)=\|u\|_{C^{4}}+|p|+\frac{1}{|\mu|} .
$$

In order to obtain a result analogous to the global Hopf bifurcation theorem of [1], we show that $\nu$ can actually become unbounded on $C(1,1)$ if and only if $\bar{\nu}$ is unbounded on $C(1,1)$.

THEOREM 4.2. If $(\mathbf{F})$ is satisfied and $u F_{u}(u) \geq 0$, then $C(1,1)$ contains a pathconnected $\bar{\nu}$-unbounded subset.

Proof. Suppose that $\nu$ is unbounded on $C(1,1)$ but that $\bar{\nu}$ is bounded on this set; it follows that there is a sequence $\left(u_{n}, p_{n}, \mu_{n}\right) \in C(1,1)$ such that $\left\|u_{n}\right\|_{C^{4}}+\left|p_{n}\right|$ is bounded, $u_{n} \neq 0$ for each $n$, and $\left|\mu_{n}\right| \rightarrow \infty$. However, since

$$
\begin{aligned}
\int_{0}^{2 \pi} p \mu^{2}\left(u^{\prime \prime}\right)^{2} d t \geq \frac{1}{2 \pi} \int_{0}^{2 \pi} p \mu^{2}\left(u^{\prime}\right)^{2} & =\frac{1}{2 \pi}\left(\int_{0}^{2 \pi} \mu^{4}\left(u^{\prime \prime}\right)^{2}+u F_{u}(u) d t\right) \\
& \geq \frac{1}{2 \pi} \int_{0}^{2 \pi} \mu^{4}\left(u^{\prime \prime}\right)^{2} d t
\end{aligned}
$$

holds for any nontrivial solution in $C(1,1)$ by the Poincaré inequality, it follows that $\mu_{n}^{2} \leq 2 \pi p_{n}$, which is a contradiction. Therefore, by Lemma 4.1, $\nu$ is unbounded on a path-connected subset of $C(1,1)$, and the above contradiction implies that $\bar{\nu}$ must also be unbounded on this set.

This theorem represents a partial global trichotomy for bifurcations of periodic orbits of (1.1), which says that the solution continuum $C(1,1)$ either has an unbounded sequence of orbits in phase-space or is unbounded with respect to either the parameter $(p)$ or with the period (as occurs in the blue-sky bifurcation [13]). Unfortunately, due to assumption (v) of Theorem 4.1, it has not proven possible to use the same techniques to study the global existence properties of the branches $C(n, m)$ for $n>1$.

5. Local secondary fold bifurcations. Another advantage of the approach taken in this paper as opposed to the shooting methods previously used in $[35,36]$ is that we can investigate the geometry of each bifurcating continuum by introducing an unfolding parameter, $\epsilon$, into (3.1)-(3.2). We will now show that degeneracies present in (3.1)-(3.2) at $\epsilon=0$ can unfold to give secondary fold bifurcations along the bifurcation branch when $\epsilon \neq 0$.

To illustrate this we shall consider (1.1) for the particular case given in (1.3). This has been studied in [19] (see also [12] for an asymptotic analysis of this problem using multiple scale techniques) as a model for an elastic rock layer on a restiffening foundation, with corresponding $\mathbb{Z}_{2}$-symmetric ODE

$$
M_{\epsilon}(u, p, \mu) \equiv \mu^{4} u^{\prime \prime \prime \prime}+p \mu^{2} u^{\prime \prime}+u-\epsilon\left(u^{3}-u^{5}\right)
$$

and even Hamiltonian

$$
H_{\epsilon}(u, p, \mu) \equiv \mu^{4} u^{\prime} u^{\prime \prime \prime}-\frac{1}{2} \mu^{4} u^{\prime \prime 2}+\frac{1}{2} p \mu^{2} u^{\prime 2}+\frac{1}{2} u^{2}-\epsilon\left(\frac{1}{4} u^{4}-\frac{1}{6} u^{6}\right) .
$$


As in the proof of Theorem 2.2, and therefore also in Theorem 3.1, we can obtain a local representation of the bifurcating branch of the zero-Hamiltonian problem associated with (5.1) from the bifurcation point $p=2$ in the form $u_{\epsilon}(\beta)=\beta\left(k+\rho_{\epsilon}(\beta)\right)$, where $\rho_{\epsilon}(\beta)=O(\beta)$ for fixed $\epsilon$ and is an analytic function of both $\beta$ and $\epsilon$ near $(\beta, \epsilon)=(0,0)$; here $k(t)=\cos (t)$. We now proceed with a calculation to find the Taylor expansion of $\rho_{\epsilon}(\beta)$ in order to determine the local geometry of the set of branches $C_{\epsilon}(1,1)$. Throughout this section we shall write

$$
r_{\epsilon}(\beta)=\beta \rho_{\epsilon}(\beta) .
$$

It is important to note that the existence of bifurcating solutions for this problem close to $p=2$, as determined by Theorem 3.1, does not depend upon the value of $\epsilon$. Indeed, using the implicit function theorem we simply find that for each $\epsilon$ sufficiently small and for suitable $m, n$, there is a bifurcating branch from $(p, \mu)=\left(p_{n, m}, \mu_{n, m}\right)$ and this branch (that is, the local parametric representation of this branch) varies analytically with $\epsilon$. This property also holds at $\epsilon=0$, where the branches are pairs of lines. We also note that since $F_{\epsilon}(u)$ is an even function of $u$ for each $\epsilon, r_{\epsilon}(\cdot)$ is odd and $p_{\epsilon}(\cdot)$ and $\mu_{\epsilon}(\cdot)$ are even functions, forming a pitchfork bifurcation at $p=2$.

We start our analysis by listing the Fréchet derivatives of the operator $M_{\epsilon}$ :

D1. $d_{u} M_{\epsilon}(u, p, \mu)[h]=\mu^{4} h^{\prime \prime \prime \prime}+p \mu^{2} h^{\prime \prime}+h-\epsilon\left(3 u^{2}-5 u^{4}\right) h$,

D2. $d_{u}^{2} M_{\epsilon}(u, p, \mu)\left[h_{1}, h_{2}\right]=-\epsilon h_{1} h_{2}\left(6 u-20 u^{3}\right)$,

D3. $d_{u}^{3} M_{\epsilon}(u, p, \mu)\left[h_{1}, h_{2}, h_{3}\right]=-\epsilon h_{1} h_{2} h_{3}\left(6-60 u^{2}\right)$,

D4. $d_{u}^{4} M_{\epsilon}(u, p, \mu)\left[h_{1}, h_{2}, h_{3}, h_{4}\right]=120 \epsilon v h_{1} h_{2} h_{3} h_{4}$,

D5. $d_{u}^{5} M_{\epsilon}(u, p, \mu)\left[h_{1}, h_{2}, h_{3}, h_{4}, h_{5}\right]=120 \epsilon h_{1} h_{2} h_{3} h_{4} h_{5}$, where $h_{i} \in X_{e}$ for each $i=1, \ldots, 5$.

We denote the first derivative of $M_{\epsilon}(u, p, \mu)$ evaluated on the trivial solution branch $u=0$ by $L \equiv d_{u} M_{\epsilon}(0,2,1)$; this operator is independent of $\epsilon$. Suppose further that $P$ is the projection of $Y_{e}$ onto $\operatorname{ran}(L)=\langle k\rangle^{\perp}$ along $\langle k\rangle$; now define

$$
L(p, \mu) \equiv d_{u} M_{\epsilon}(0, p, \mu) .
$$

We can solve the projected differential equation $P \circ N_{\epsilon}(\beta k+r, p, \mu)=0$ for some function $r=r_{\epsilon}(\beta, p, \mu)$ near $(\beta, p, \mu, \epsilon)=(0,2,1 ; 0)$ using the implicit function theorem (we refer to the proof of Theorem 2.2 for details). From the uniqueness properties of the implicit function theorem if follows that $r_{\epsilon}(0, p, \mu) \equiv 0$, and if we repeatedly differentiate the identity $P M_{\epsilon}\left(\beta k+r_{\epsilon}(\beta, p, \mu), p, \mu\right)=0$ with respect to $\beta$, then we shall obtain the Taylor coefficients of $r_{\epsilon}$. This is a tedious exercise, so we omit the details, but one eventually obtains

R1. $P\left(d_{u} M_{\epsilon}\left[k+d_{\beta} r\right]\right) \equiv 0$,

R2. $P\left(d_{u}^{2} M_{\epsilon}\left[k+d_{\beta} r, k+d_{\beta} r\right]+d_{u} M_{\epsilon}\left[d_{\beta}^{2} r\right]\right) \equiv 0$,

R3. $P\left(d_{u}^{3} M_{\epsilon}\left[k+d_{\beta} r\right]^{3}+2 d_{u}^{2} M_{\epsilon}\left[d_{\beta}^{2} r, k+d_{\beta} r\right]+d_{u}^{2} M_{\epsilon}\left[d_{\beta}^{2} r, k+d_{\beta} r\right]+d_{u} M_{\epsilon}\left[d_{\beta}^{3} r\right]\right) \equiv 0$,

R4. $P\left(d_{u}^{4} M_{\epsilon}\left[k+d_{\beta} r\right]^{4}+6 d_{u}^{3} M_{\epsilon}\left[d_{\beta}^{2} r, k+d_{\beta} r, k+d_{\beta} r\right]+3 d_{u}^{2} M_{\epsilon}\left[d_{\beta}^{3} r, k+d_{\beta} r\right]+\right.$ $\left.3 d_{u}^{2} M_{\epsilon}\left[d_{\beta}^{2} r, d_{\beta}^{2} r\right]+d_{u} M_{\epsilon}\left[d_{\beta}^{4} r\right]+d_{u}^{2} M_{\epsilon}\left[k+d_{\beta} r, d_{\beta}^{3} r\right]\right) \equiv 0$,

R5. $P\left(d_{u}^{5} M_{\epsilon}\left[k+d_{\beta} r\right]^{5}+10 d_{u}^{4} M_{\epsilon}\left[d_{\beta}^{2} r,\left[k+d_{\beta}^{3} r\right]^{3}\right]+10 d_{u}^{3} M_{\epsilon}\left[d_{\beta}^{3} r, k+d_{\beta} r, k+d_{\beta} r\right]+\right.$ $\left.3 d_{u}^{2} M_{\epsilon}\left[d_{\beta}^{4} r, d_{\beta} r\right]+10 d_{u}^{2} M_{\epsilon}\left[d_{\beta}^{3} r, d_{\beta}^{2} r\right]+2 d_{u}^{2} M_{\epsilon}\left[d_{\beta}^{4} r, d_{\beta} r\right]+d_{u} M_{\epsilon}\left[d_{\beta}^{5} r\right]\right) \equiv 0$.

Evaluating these expressions at $\beta=0$, that is, $u=r_{\epsilon}(0, p, \mu)=0$, yields the following information. From R1 we have $P L(p, \mu)\left[k+d_{\beta} r(0, p, \mu ; \epsilon)\right]=0$, and because $L(p, \mu) k=\left(\mu^{4}-p \mu^{2}+1\right) k \in\langle k\rangle$ we have $d_{\beta} r_{\epsilon}(0, p, \mu) \equiv 0$. The expression $d_{\beta}^{2} r_{\epsilon}(0, p, \mu)=0$ then follows from R2. Also, R3 gives

$$
P L(p, \mu) d_{\beta}^{3} r_{\epsilon}(0, p, \mu)=6 \epsilon P\left(k^{3}\right),
$$


so that the third derivative of $r_{\epsilon}$ is not zero in general at $(u, p, \mu ; \epsilon)=(0,2,1 ; \epsilon)$, but $d_{\beta}^{3} r_{\epsilon}(0, p, \mu)$ is seen to provide an $O(\epsilon)$ contribution to the Taylor expansion of $r_{\epsilon}$. Using Taylor's theorem to expand $r_{\epsilon}(\beta, p, \mu)$ with respect to $\beta$ and using the symmetry properties of $r_{\epsilon}$ (it is odd with respect to $\beta$ ), we may write $r_{\epsilon}(\beta, p, \mu)=$ $\frac{\beta^{3}}{6} R_{\epsilon}^{1}(p, \mu)+O\left(\beta^{5}\right)$ for some operator $R_{\epsilon}^{1}(p, \mu)$ with range in $\langle k\rangle^{\perp}$.

We can determine $R_{\epsilon}^{1}$ as follows. In (5.3) seek an even Fourier series solution which is also orthogonal to $k$ in $X_{e}$ of the form $R_{\epsilon}^{1}(p, \mu)=\sum_{j=2}^{\infty} a_{j} \cos (j t)$, where the coefficients $a_{j}$ remain to be determined. Since $k^{3}(t)=\frac{1}{4}(\cos (3 t)+3 \cos (t))$, it follows that the only nonzero coefficient is $a_{3}$ and

$$
R_{\epsilon}^{1}(p, \mu)=\frac{3 \epsilon}{2} \frac{\cos (3 \cdot)}{81 \mu^{4}-9 p \mu^{2}+1} .
$$

Using R4 and setting $\beta=0$, we find $d_{\beta}^{4} r_{\epsilon}(0, p, \mu) \equiv 0$, which of course also follows from symmetry. We may evaluate $d_{\beta}^{5} r_{\epsilon}(0, p, \mu)$ from $\mathrm{R} 5$, which simplifies to give

$$
P\left(d_{u}^{5} M_{\epsilon}[k]^{5}+10 d_{u}^{3} M_{\epsilon}\left[k, k, d_{\beta}^{3} r_{\epsilon}\right]+d_{u} M_{\epsilon}\left[d_{\beta}^{5} r_{\epsilon}\right]\right) \equiv 0 .
$$

To find $d_{\beta}^{5} r_{\epsilon}(0, p, \mu)$ we solve the following linear equation for $w \in X_{e} \cap\langle k\rangle^{\perp}$,

$$
\mu^{4} w^{\prime \prime \prime \prime}+p \mu^{2} w^{\prime \prime}+w+P\left[60 k^{2} \frac{3 \epsilon^{2}}{2} \frac{\cos (3 \cdot)}{81 \mu^{4}-9 p \mu^{2}+1}+120 \epsilon k^{5}\right]=0,
$$

and then $d_{\beta}^{5} r_{e}(0, p, \mu)=w$. Since $k(t)^{5}=\frac{1}{16}(\cos (5 t)+5 \cos (3 t)+10 \cos (t)), k(t)^{2}=$ $\frac{1}{2}(1+\cos (2 t))$, and $\cos (3 t) \cos (2 t)=\frac{1}{2}(\cos (5 t)+\cos (t))$, we also solve (5.4) using a Fourier series expansion. Accordingly, taking $w(t)=\sum_{j=2}^{\infty} w_{j} \cos (j t)$, we find that all the coefficients $w_{j}$ are zero, except when $j=3$ or $j=5$. In these cases

$$
\left(81 \mu^{4}-9 p \mu^{2}+1\right) w_{3}+150 \epsilon-\frac{45 \epsilon^{2}}{81 \mu^{4}-9 p \mu^{2}+1}=0
$$

and

$$
\left(625 \mu^{4}-25 p \mu^{2}+1\right) w_{5}+30 \epsilon-\frac{90 \epsilon^{2}}{2\left(81 \mu^{4}-9 p \mu^{2}+1\right)}=0 .
$$

It follows that $r_{\epsilon}(\beta, p, \mu)=\frac{\beta^{3}}{6} R_{\epsilon}^{1}(p, \mu)+\frac{\beta^{5}}{120} R_{\epsilon}^{2}(p, \mu)+\frac{\beta^{7}}{720} d_{\beta}^{7} r_{\epsilon}(0, p, \mu)+O\left(\epsilon^{2} \beta^{3}\right)$, where

$R_{\epsilon}^{2}(p, \mu)=-\epsilon\left(\frac{150}{4\left(81 \mu^{4}-9 p \mu^{2}+1\right)} \cos (3 \cdot)+\frac{30}{4\left(625 \mu^{4}-25 p \mu^{2}+1\right)} \cos (5 \cdot)\right)+O\left(\epsilon^{2}\right)$.

One can show by further differentiating that $d_{\beta}^{6} r_{\epsilon}(0, p, \mu) \equiv 0$, as we expect from symmetry, and the equation which determines $d_{\beta}^{7} r_{\epsilon}(0, p, \mu)$ shows this term to be of order $O\left(\epsilon^{2}\right)$. Higher derivatives of $r_{\epsilon}$ will also be of order $O\left(\epsilon^{2}\right)$.

Now $p_{\epsilon}(\beta)$ and $\mu_{\epsilon}(\beta)$ are even functions of $\beta$, and applying the zero-Hamiltonian constraint gives

$$
\mu^{4} \delta\left(\left(k+\rho_{\epsilon}\right)^{\prime \prime 2}\right)=\delta\left(\left(k+\rho_{\epsilon}\right)^{2}-\frac{1}{2} \epsilon \beta^{2}\left(k+\rho_{\epsilon}\right)^{4}+\frac{1}{3} \epsilon \beta^{4}\left(k+\rho_{\epsilon}\right)^{6}\right),
$$


using a prime to denote $\frac{d}{d t}$. Seeking an expansion of the bifurcation branch about $(\epsilon, \beta)=(0,0)$, we write

$$
p_{\epsilon}(\beta)=2+\epsilon\left(P_{1} \beta^{2}+P_{2} \beta^{4}\right)+O\left(\epsilon^{2}\right)
$$

and

$$
\mu_{\epsilon}(\beta)=1+\epsilon\left(\omega_{1} \beta^{2}+\omega_{2} \beta^{4}\right)+O\left(\epsilon^{2}\right),
$$

where $P_{1}, P_{2}, \omega_{1}$, and $\omega_{2}$ are real numbers to be determined. The highest power of $\beta$ which exists in these expansions at $O(\epsilon)$ is the quartic because of the Taylor series we have found for $r_{\epsilon}(\beta)$. This is clear from (5.5), which contains terms of order $\epsilon \beta^{2}$ and $\epsilon \beta^{4}$ but not $\epsilon \beta^{6}$ or higher.

To determine $\omega_{1}$ and $\omega_{2}$ we substitute the expressions for $\rho_{\epsilon}=\frac{r_{e}}{\beta}$ and $\mu_{\epsilon}$ into (5.5). Using $\delta(\rho)=\left(\frac{1}{256} \beta^{2}-\frac{23}{4608} \beta^{4}\right) \epsilon+O\left(\epsilon^{2}\right)$ and $\delta\left(\rho^{\prime \prime}\right)=\left(-\frac{9}{256} \beta^{2}+\frac{215}{4608} \beta^{4}\right) \epsilon+O\left(\epsilon^{2}\right)$, we then find $\frac{1}{\left(81 \mu^{4}-9 p \mu^{2}+1\right)}=\frac{1}{64}+O(\epsilon)$ and $\frac{1}{\left(625 \mu^{4}-25 p \mu^{2}+1\right)}=\frac{1}{576}+O(\epsilon)$. Setting $v=\beta(k+\rho) \in\langle k\rangle \oplus\langle k\rangle^{\perp}$ in (5.1) and projecting the result onto the span of $k(t)=$ $\cos (t)$, we obtain

$$
\mu^{4}-p \mu^{2}+1-\epsilon \beta^{2} \frac{1}{\pi} \int_{0}^{2 \pi} k\left((k+\rho)^{3}-\beta^{2}(k+\rho)^{5}\right) d t=0 .
$$

We now use this information to equate coefficients at the appropriate orders to find

$$
P_{1}=-\frac{3}{4}, \quad P_{2}=\frac{5}{8}, \quad \omega_{1}=\frac{-9}{64}, \quad \text { and } \omega_{2}=\frac{5}{48} .
$$

5.1. Conditions for a fold bifurcation. The solution branch $C_{\epsilon}(1,1)$ determined above, which branches from $p=2$, can be continued from bifurcation in $p$ for $p<2$, or in $\mu$ for $\mu<1$. When considered as a function of $p$, the branch has a fold bifurcation at some point, which we label $p_{F}$, and the same behavior is observed when the branch is continued in $\mu$. The numerical calculations presented in the next section also indicate that the solution branch is restricted to the parameter range $p>p_{F}$ and $\mu>\mu_{F}$, although we have no proof of this claim.

We can now prove the following theorem.

THEOREM 5.1. There is a neighborhood $I \subset \mathbb{R}$ of zero such that if $\epsilon \in I$, the zero-Hamiltonian branch $C_{\epsilon}(1,1)$ associated with $(5.1)$, which bifurcates from $p=2$ at $\mu=1$, has a fold which occurs with respect to $p$ at

$$
p_{F}(\epsilon)=2-\frac{9}{40} \epsilon+O\left(\epsilon^{2}\right) .
$$

There is also a fold in $C_{\epsilon}(1,1)$ with respect to $\mu$ which occurs at

$$
\mu_{F}(\epsilon)=1-\frac{243}{5120} \epsilon+O\left(\epsilon^{2}\right) .
$$

Proof. Using (5.6), (5.7), and (5.9), a fold bifurcation with respect to $p$ occurs on the $C_{\epsilon}(1,1)$ branch when the conditions $d_{\beta} p_{\epsilon}(\beta)=0$ and $d_{\beta \beta}^{2} p_{\epsilon}(\beta) \neq 0$ are met. Applying the implicit function theorem when $\epsilon \neq 0$, these conditions are satisfied when $\beta^{2}=\beta_{F}^{2} \equiv 3 / 5+\mathcal{O}(\epsilon)$, giving (5.10).

Similarly, a fold bifurcation with respect to $\mu$ occurs on the branch $C_{\epsilon}(1,1)$ when $d_{\beta} \mu_{\epsilon}(\beta)=0$ and $d_{\beta \beta}^{2} \mu_{\epsilon}(\beta) \neq 0$, and, provided $\epsilon \neq 0$, these conditions are satisfied when $\beta^{2}=27 / 40+\mathcal{O}(\epsilon)$, giving (5.11). 


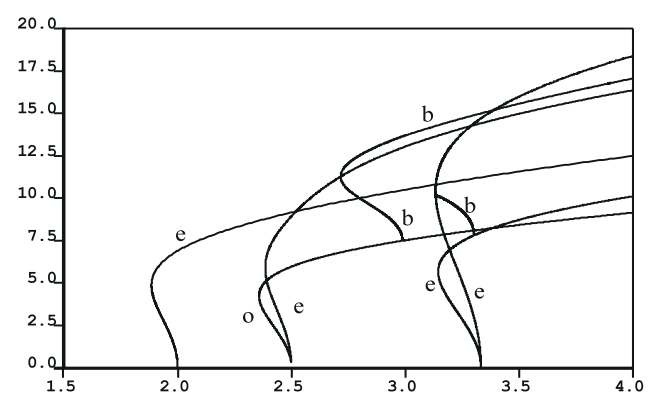

FIG. 6.1. Bifurcations of zero-Hamiltonian periodic solutions from $p=2, p=2 \frac{1}{2}$, and $p=3 \frac{1}{3}$, with $p$ plotted horizontally against $\left\|u^{\prime}\right\|_{L^{\infty}}$ vertically; $e$ are even solutions, o are odd solutions, and $b$ are solutions with broken symmetry.

\section{Numerical computations.}

6.1. Preliminaries. We now describe a series of numerical calculations to determine solutions of the unscaled differential equation (1.1) with the restiffening foundation whose primary solution branch was studied in the previous section:

$$
u^{\prime \prime \prime \prime}+p u^{\prime \prime}+u-\epsilon\left(u^{3}-u^{5}\right)=0 .
$$

We augment this with the periodic boundary conditions $u(0)=u(T), u^{\prime}(0)=u^{\prime}(T)$, and $u^{\prime \prime \prime}(0)=u^{\prime \prime \prime}(T)$ and specify the phase by requiring $u^{\prime}(0)=0$. Finally, we impose the constraint that the Hamiltonian is zero, so that

$$
u^{\prime \prime}(0)= \pm \sqrt{2\left(u(0)^{2} / 2-\epsilon\left(u(0)^{4} / 4-u(0)^{6} / 6\right)\right.},
$$

and we set $\epsilon=1 / 2$ for the purposes of computation.

In (6.2) the positive root corresponds to the solution which is tangential to the rescaled eigensolution $e_{-}(x) \equiv n \cos (x \sqrt{m / n})-m \cos (x \sqrt{n / m})$ at the bifurcation point $(u, p, \mu)=\left(0, p_{n, m}, \mu_{n, m}\right)$, whereas the negative root corresponds to the solution which is tangential to the eigensolution $e_{+}(x) \equiv n \cos (x \sqrt{m / n})+m \cos (x \sqrt{n / m})$. In order to follow the solution branches in $p$ and to detect fold bifurcations, the collocation-based code AUTO [14] was used.

6.2. Calculation of the solution branches. We now illustrate three cases regarding the bifurcation of solutions of $(6.1)$ : the case $(n, m)=(1,1)$, for which there is a unique bifurcating branch; the case $(n, m)=(2,1)$, with $p_{2,1}=2 \frac{1}{2}$ and $\mu_{2,1}=\frac{1}{\sqrt{2}}$; and $(n, m)=(3,1)$, for which $p_{3,1}=3 \frac{1}{3}$ and $\mu_{1,3}=\frac{1}{\sqrt{3}}$. Broadly speaking, higher values of $n$ and $m$ lead to similar solution branches.

Figure 6.1 shows the bifurcation branches which are proven to exist in Theorems 3.1 and 3.3 , with $p$ plotted against $\left\|u^{\prime}\right\|_{L^{\infty}}$. The $(1,1)$ branch has the form described in Theorem 5.1, and for the $p=2 \frac{1}{2}$ and $p=3 \frac{1}{3}$ cases one sees a similar geometry in that the branches initially bifurcate to the left, have fold bifurcations, and then persist for all values of $p$ to the right of the fold point.

The following comments are in order regarding Figure 6.1 and the three points $p=2,2 \frac{1}{2}$, and $3 \frac{1}{3}$. Due to the $\mathbb{Z}_{2}$-symmetry of $(6.1)$ and of the symmetry properties of the eigenfunctions when $(n, m)=(2,1)$, if $u(t)$ is one even solution on the $(2,1)$ branch, then so too is $-u(t+T / 2)$. Therefore, in order to obtain a second distinct periodic solution, we apply Theorem 3.5 to give the existence of two branches of odd 


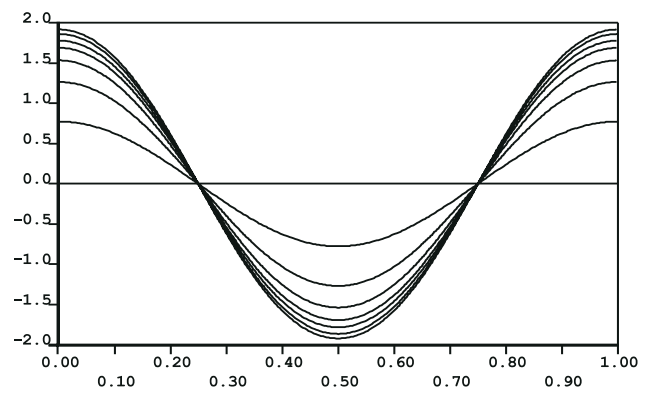

FIG. 6.2. Solutions $(u(s)$ for $0 \leq s \leq 1)$ bifurcating from $p=2$, away from the bifurcation point. In accordance with Theorem 2.2 these are even about zero and odd about one-quarter.
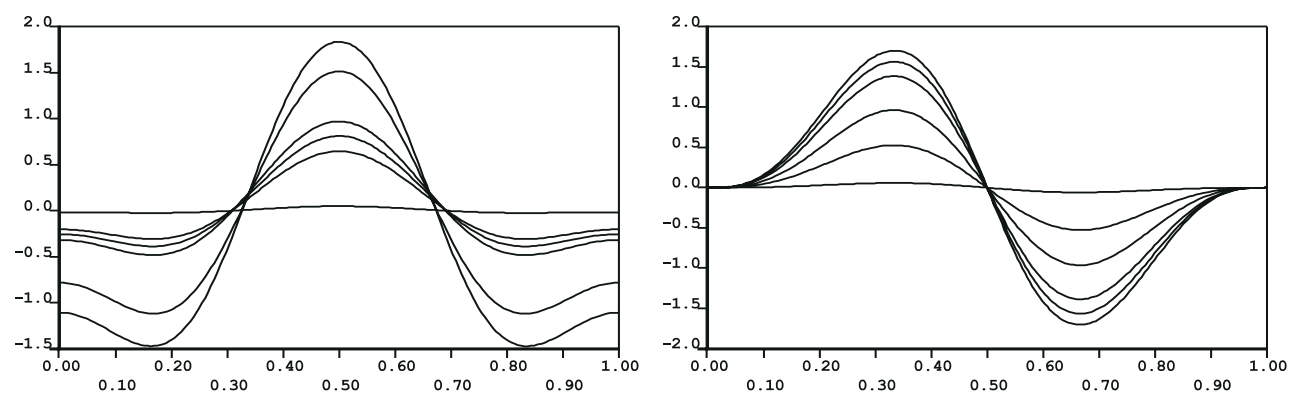

FIG. 6.3. (left) Even solutions $(u(s)$ for $0 \leq s \leq 1)$ bifurcating from $p=2 \frac{1}{2}$, away from the bifurcation point. (right) Odd solutions bifurcating from $p=2 \frac{1}{2}$.
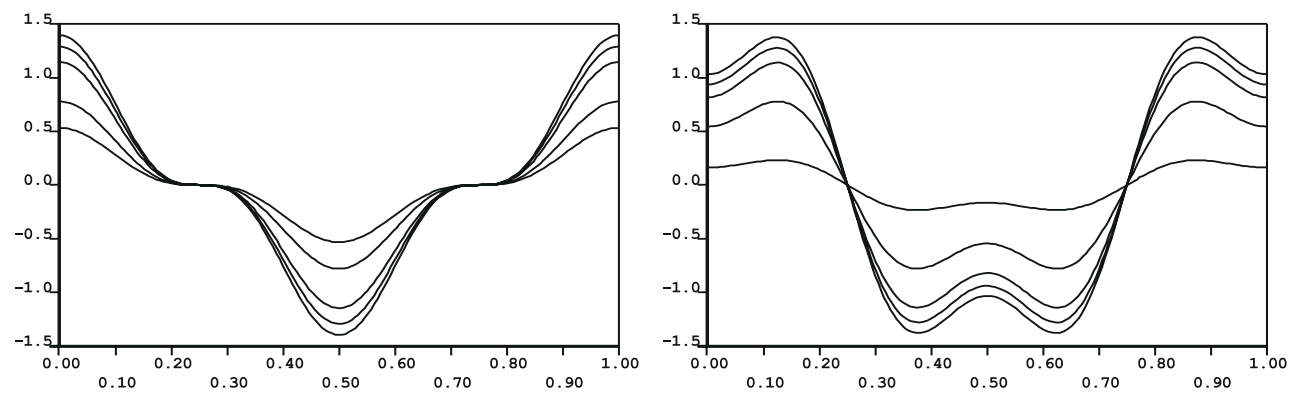

FIG. 6.4. Solutions $(u(s)$ for $0 \leq s \leq 1)$ bifurcating from $p=3 \frac{1}{3}$, away from the bifurcation point.

solutions. Again, one of these branches of odd solutions can be obtained from the other by symmetry, and we therefore have plotted one of each even and odd branch in Figure 6.1. The solutions on this branch are shown in Figure 6.3.

Figures $6.2,6.3$, and 6.4 each show several solutions chosen from Figure 6.1 on the branches which connect to $p=2, p=2 \frac{1}{2}$, and $p=3 \frac{1}{3}$, respectively, although the domain of each solution has been normalized to unity. (The information regarding the period of the solutions is given in Figure 6.5.) If we examine Figure 6.4, we notice that each of the solutions is even about zero and odd about one quarter. Consequently, the two branches of the solutions shown are in fact identical, up to a shift and a reflection, to the odd solutions which are obtained using Theorem 3.5.

Since $F(u)$ and $u F_{u}(u)$ are both positive for nonzero $u$ when $\epsilon=1 / 2$, the global 


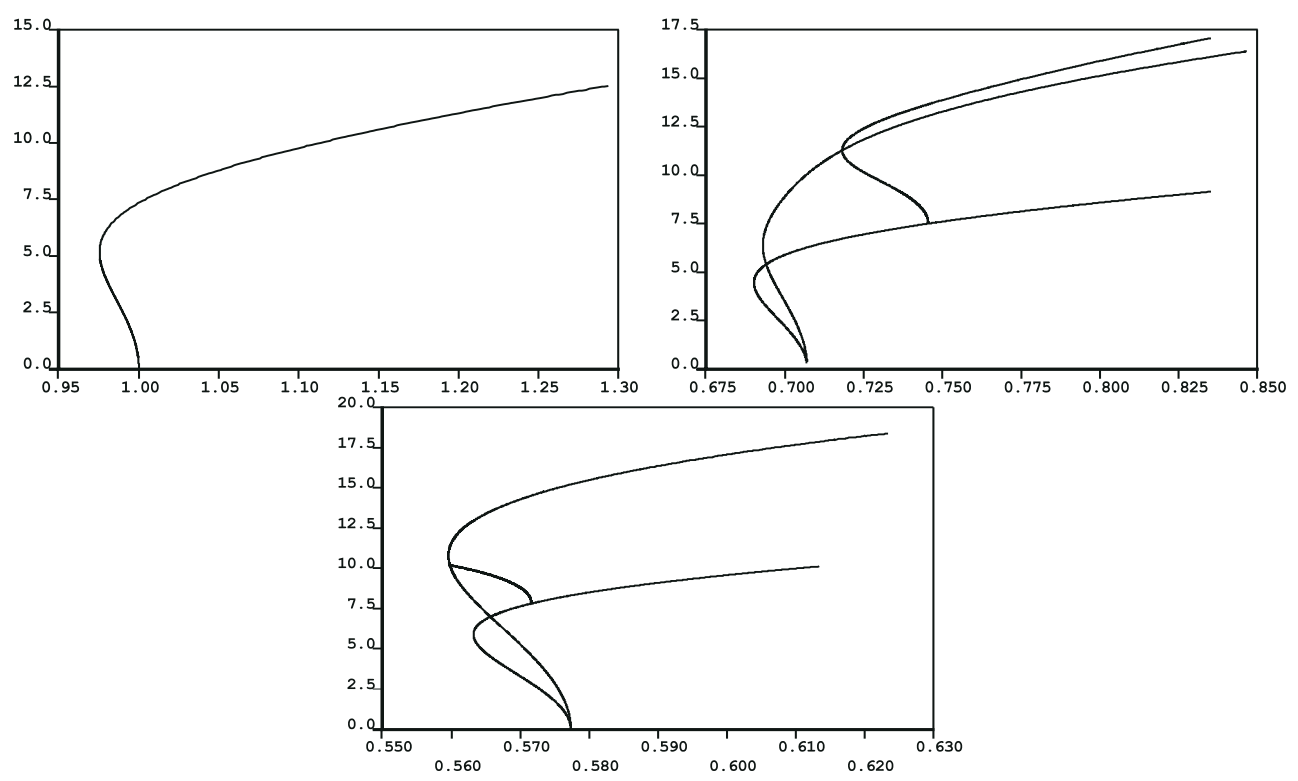

FIG. 6.5. Period of solutions from Figure $6.1((1,1)$ branch is top-left, $(2,1)$ top-right, $(3,1)$ bottom) with $\mu=2 \pi /$ period plotted horizontally and $\left\|u^{\prime}\right\|_{L^{\infty}}$ vertically.

bifurcation theorem (Theorem 4.2$)$ applies to the $(1,1)$ branch, and the nodal properties are preserved along the resulting global branch in accordance with Theorems 3.6, 3.7, and 3.8. This is illustrated in each of Figures 6.2, 6.3, and 6.4.

Finally, note that the $(1,1)$ branch in Figure 6.1 appears to have no further bifurcations, whereas the $(2,1)$ and $(3,1)$ branches both have symmetry-breaking secondary bifurcation points. What is interesting about the resulting branches of unsymmetric solutions is that they form connections between the $(2,1)$ and $(3,1)$ branches. This indicates that it would be futile to seek generalizations of the results of section 3.5 to include the space of all periodic zero-Hamiltonian solutions of (1.1) and that the disjointness properties of the solution branches obtained in this paper are peculiar to spaces of symmetric solutions.

\section{REFERENCES}

[1] J. C. Alexander And J. A. Yorke, Global bifurcations of periodic solutions, Amer. J. Math., 100 (1978), pp. 263-292.

[2] A. Ambrosetti and G. Prodi, A Primer of Nonlinear Analysis, Cambridge Stud. Adv. Math. 34, Cambridge University Press, Cambridge, UK, 1992.

[3] C. J. Amick And J. F. Toland, Global uniqueness of homoclinic orbits for a class of fourthorder equations, Z. Angew. Math. Phys., 43 (1992), pp. 591-597.

[4] M. Boughariou, Closed orbits of Hamiltonian systems on non-compact prescribed energy surfaces, Discrete Contin. Dynam. Systems, 9 (2003), pp. 603-616.

[5] R. F. Brown, A Topological Introduction to Nonlinear Analysis, Birkhäuser Boston, Cambridge, MA, 1993.

[6] B. Buffoni, E. Dancer, and J. Toland, A variational theory of Stokes waves and their sub-harmonic bifurcations, Arch. Ration. Mech. Anal., 2001, preprint; available online from http://www.maths.bath.ac.uk/MATHEMATICS/preprints.html — find preprint maths9801.

[7] B. Buffoni And J. F. Toland, Global existence of homoclinic and periodic orbits for a class of autonomous Hamiltonian systems, J. Differential Equations, 118 (1995), pp. 104-120. 
[8] A. R. Champneys, Homoclinic orbits in reversible systems and their applications in mechanics, fluids and optics, Phys. D, 112 (1998), pp. 158-186.

[9] A. R. Champneys and J. M. T. Thompson, A multiplicity of localized buckling modes for twisted rod equations, Proc. Royal Soc. Ser. A, 452 (1996), pp. 2467-2491.

[10] J. V. Chaparova, L. A. Peletier, and S. A. Tersian, Existence and nonexistence of nontrivial solutions of semilinar fourth and sixth-order differential equations, preprint, from http://www.math.leidenuniv.nl/ peletier.

[11] S. N. ChOw And J. K. Hale, Methods of Bifurcation Theory, Springer-Verlag, New York, 1982.

[12] C. J. Budd, G. W. Hunt, And R. A. Kuske, Cellular buckling close to Maxwell load, Proc. Roy. Soc. London Ser. A, 457 (2001), pp. 2935-2964.

[13] R. L. Devaney, Blue sky catastrophes in reversible and Hamiltonian systems, Indiana Univ. Math. J., 26 (1977), pp. 247-263.

[14] E. J. Doedel, A. R. Champneys, T. F. Fairgrieve, Y. A. Kuznetsov, B. Sandstede, and X.-J. WANG, AUTO97: Continuation and Bifurcation Software for Ordinary Differential Equations, Tech. report, Department of Computer Science, Concordia University, Montreal, Canada, 1997; available by FTP from ftp.cs.concordia.ca in directory pub/doedel/auto.

[15] W. Eckhaus, Singular perturbations of homoclinic orbits in $\mathbb{R}^{4}$, SIAM J. Math. Anal., 23 (1992), pp. 1269-1290.

[16] M. Feckan and V. Rothos, Bifurcations of periodics from homoclinics in singular ODE: Applications to discretizations of travelling waves of PDE, Comm. Pure Appl. Anal., 1 (2002), pp. 475-483.

[17] H. Hofer And J. F. Toland, On the existence of homoclinic, heteroclinic and periodic orbits for a class of indefinite Hamiltonian systems, Math. Ann., 12 (1984), pp. 387-403.

[18] G. W. Hunt, G. J. Lord, And A. R. Champneys, Homoclinic and heteroclinic orbits underlying the post-buckling of axially compressed cylindrical shells, Comput. Methods Mech. Engrg., 170 (1999), pp. 239-251.

[19] G. W. Hunt, M. A. Peletier, A. R. Champneys, P. D. Woods, M. A. Wadee, C. J. Budd, AND G. J. LORD, Cellular buckling in long structures, Nonlinear Dynamics, 1 (2000), pp. 329 .

[20] W. D. Kalies, J. Kwapisz, J. van den Berg, and R. van Der Vorst, Homotopy classes for stable periodic and chaotic patterns in fourth-order Hamiltonian systems, Comm. Math. Phys., 214 (2000), pp. 573-592.

[21] Y. S. Kivshar, A. R. Champneys, D. CaI, And A. R. Bishop, Multiple states of intrinsic localised modes, Phys. Rev. B, 58 (1988), pp. 5423-5428.

[22] J. Knobloch and A. VAnderbauwhede, A general reduction method for periodic solutions in conservative and reversible systems, Dyn. Difference Equations, 8 (1996), pp. 71-102.

[23] C. LAZZARI, Symmetries and exponential smallness of bifurcation functions of a class of singular, reversible systems, Nonlinear Anal., 33 (1998), pp. 759-772.

[24] V. J. Mizel, L. A. Peletier, And W. C. Troy, Periodic phases in second-order materials, Arch. Ration. Mech. Anal, 145 (1998), pp. 343-382.

[25] L. Peletier And W. Troy, Spatial patterns, higher order models in physics and mechanics, Progress in Nonlinear Differential Equations and Their Applications, Vol. 45, Birkhäuser Boston, Cambridge, MA, 2001.

[26] L. A. Peletier, A. I. Rotariu-Bruma, and W. C. Troy, Pulse-like spatial patterns described by higher-order model equations, J. Differential Equations, 150 (1998), pp. 124-187.

[27] L. A. Peletier and W. C. Troy, Chaotic spatial patterns described by the extended FisherKolmogorov equation, J. Differential Equations, 129 (1996), pp. 458-508.

[28] L. A. Peletier And W. C. Troy, Spatial patterns described by the extended FisherKolmogorov equation: Periodic solutions, SIAM J. Math. Anal., 28 (1997), pp. 1318-1354.

[29] M. A. Peletier, Non-existence and uniqueness for fourth-order Hamiltonian systems, Nonlinearity, 12 (1999), pp. 1555-1570.

[30] P. H. Rabinowitz, Periodic solutions of a Hamiltonian system on a prescribed energy surface, Differential Equations, 33 (1979), pp. 336-352.

[31] P. H. RABINOwitz, The prescribed energy problem for periodic solutions of Hamiltonian systems, Contemp. Math., 81 (1988), pp. 183-191.

[32] M. TAYlor, Partial Differential Equations: Basic Theory, Appl. Math. Sci. 1, Springer-Verlag, New York, 1996.

[33] S. Tersian and J. Chaparova, Periodic and homoclinic solutions of some semilinear sixthorder differential equations, J. Math. Anal. Appl., 272 (2002), pp. 223-239.

[34] J. F. Toland, Solitary wave solutions for a model of the two-way propagation of water waves in a channel, Math. Proc. Cambridge Philos. Soc., 90 (1981), pp. 343-360.

[35] J. B. VAN DEN BERG, The phase-plane picture for a class of fourth-order conservative differential equations, J. Differential Equations, 161 (2000), pp. 110-153. 
[36] J. B. van den Berg, L. A. Peletier, and W. C. Troy, Global branches of multi-bump periodic solutions of the Swift-Hohenberg equation, Arch. Ration. Mech. Anal, 158 (2001), pp. 91-153.

[37] A. Vanderbauwhede, Local bifurcation and symmetry, Res. Notes in Math. 75, Pitman, London, 1982.

[38] E. ZeidLer, Nonlinear Functional Analysis and Its Applications: Fixed Point Theorems, Vol. 1, Springer-Verlag, New York, 1986. 